\title{
Nature of Interactions between Epoxides in Graphene Oxide
}

Pattath D. Pancharatna, Gaurav Jhaa and Musiri M Balakrishnarajan* e-mail: mmbkr.che@pondiuni.edu.in

\section{Supplementary Information \\ Contents}

1. Figure S1-Cyclohexane isomers hosting epoxides bridges with relative energies

2. Figure S2- Construction of the isomers of $\mathrm{C} 2 \mathrm{O}$

$\begin{array}{ll}\text { 3. Figure S3- Phonon dispersion of GO nanosheets } & 10\end{array}$

4. Figure S4- Band structures of GO nanosheets 11

5. Figure S5- Density of State of GO nanosheets 12

6. Figure S6- COOP (Crystal orbital overlap population) plots of GO nanosheets 13

$\begin{array}{ll}\text { 7. Figure S7- Epoxide dimers with coordinates } & 14\end{array}$

8. Figure S8- Interaction-correlation diagram of epoxide trans dimer 17

9. Figure S9- The SALCS of the triangular ring of a sp element 18

10. Figure S10- Topology of GO isomers with coordinates 19

11. Table S1- Geometrical details of the various optimized C2O sheets 27 


\section{Nature of Interactions between Epoxides in Graphene Oxide}

\section{Supplementary Information}

Figure S1. Cyclohexane isomers hosting epoxides bridges with relative energies in parenthesis (kcal/mol).
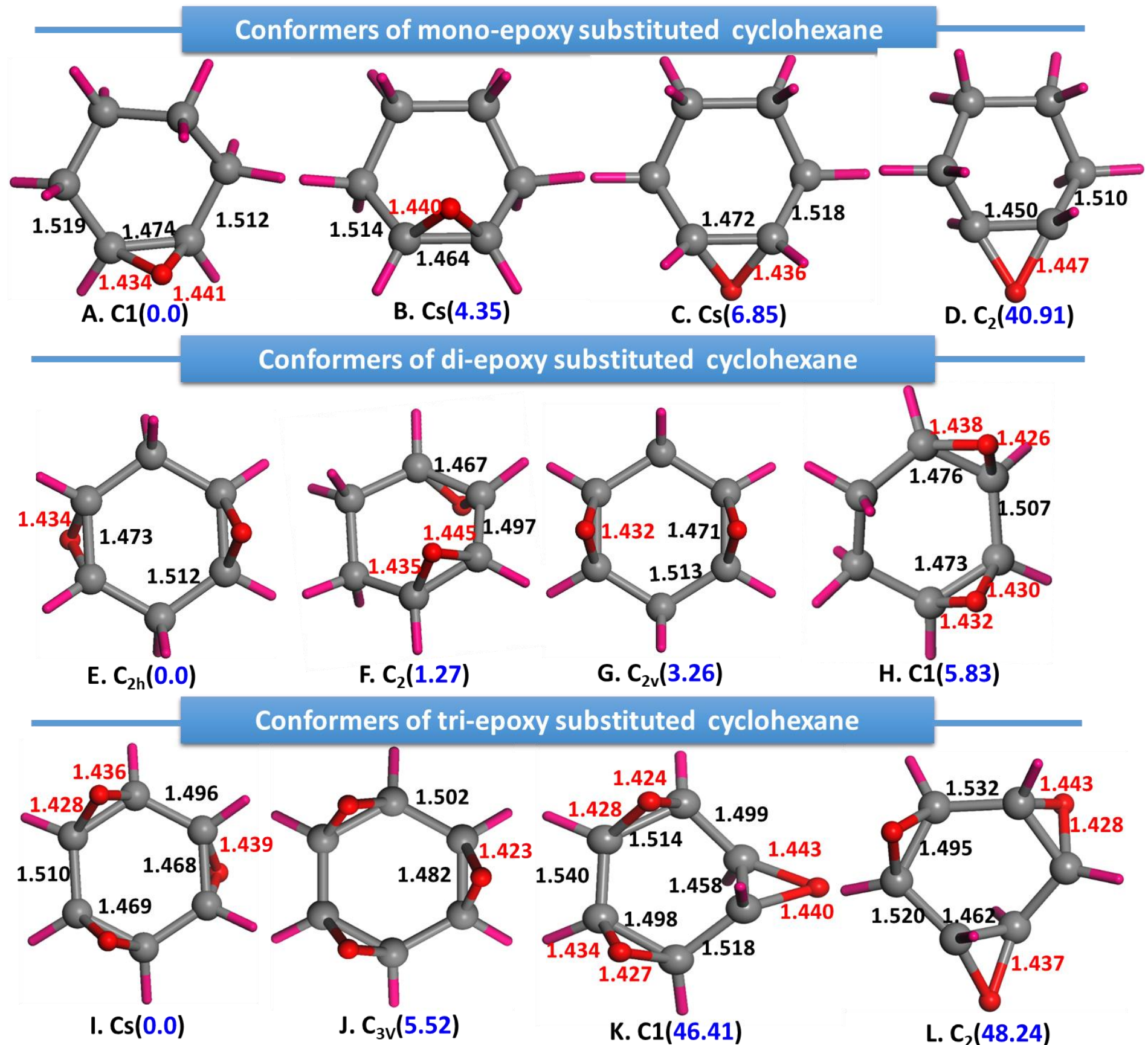
Coordinates of above Cyclohexane isomers hosting epoxides bridges:

Geometry Optimization:

Method: B3LYP; Basis Set: 6-311G(d,p)

Single Point Calculations:

Method: CCSD; Basis Set: aug-cc-Pvtz

A. $C_{1}$

\begin{tabular}{llcc}
\hline Atomic & \multicolumn{3}{c}{ Coordinates (Angstroms) } \\
Number & $X$ & $Y$ & $Z$
\end{tabular}

$1 \quad-1.919657 \quad-1.041288-0.996882$

$6 \quad-1.096245-0.630836-0.412834$

$6 \quad 1.279464 \quad-0.807709 \quad 0.439472$

$\begin{array}{lllll}6 & 0.343049 & 1.517729 & -0.035445\end{array}$

$6 \quad 1.551093 \quad 0.573648 \quad-0.169186$

$\begin{array}{lllll}6 & -0.988319 & 0.835009 & -0.295624\end{array}$

$6 \quad 0.127699-1.510545-0.293556$

$11.023731 \quad-0.701996 \quad 1.498212$

$\begin{array}{lllll}1 & 0.304300 & 1.942729 & 0.973770\end{array}$

$\begin{array}{lllll}1 & 1.794477 & 0.447349 & -1.231666\end{array}$

$\begin{array}{lllll}1 & -1.749370 & 1.442495 & -0.782672\end{array}$

$\begin{array}{llll}1 & 0.445127 & -1.789690 & -1.306632\end{array}$

$\begin{array}{llll}1 & 2.178616 & -1.428507 & 0.386832\end{array}$

$\begin{array}{lllll}1 & 0.452283 & 2.361326 & -0.724592\end{array}$

$\begin{array}{lllll}1 & 2.428348 & 1.031721 & 0.297179\end{array}$

$\begin{array}{llll}1 & -0.145540 & -2.440221 & 0.217847\end{array}$

$\begin{array}{llll}8 & -1.514095 & 0.039038 & 0.783955\end{array}$

B. $C_{s}$

\begin{tabular}{lrrr}
\hline \multicolumn{3}{l}{ Atomic } & \multicolumn{3}{c}{ Coordinates (Angstroms) } \\
Number & $X$ & $Y$ & $Z$ \\
& & & \\
\hline 1 & -0.901854 & 1.880463 & 1.260697 \\
6 & -0.442286 & 1.047276 & 0.732188 \\
6 & 0.512141 & -1.303652 & 0.778842 \\
6 & 0.512141 & -1.303652 & -0.778842 \\
6 & -0.442286 & 1.047276 & -0.732188 \\
6 & -0.442286 & -0.291329 & 1.439501 \\
1 & 1.524360 & -1.079760 & 1.121093 \\
1 & -1.474330 & -0.666323 & 1.433017 \\
1 & 0.273782 & -2.301837 & 1.156410 \\
1 & 0.273782 & -2.301837 & -1.156410 \\
1 & -0.901854 & 1.880463 & -1.260697 \\
1 & -0.168688 & -0.166976 & 2.492217 \\
1 & 1.524360 & -1.079760 & -1.121093 \\
6 & -0.442286 & -0.291329 & -1.439501
\end{tabular}



Total Energy $=-309.93242856$ a.u (DFT)

Total Energy = -309.28181292 a.u (CCSD)

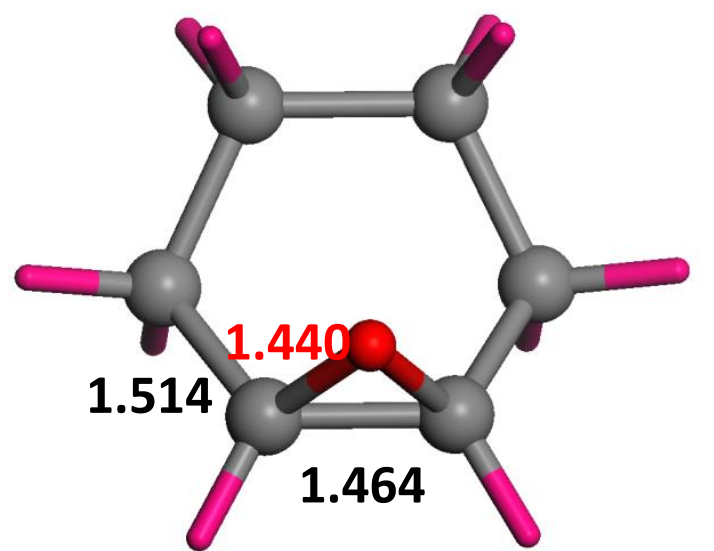

Total Energy $=-309.92542732$ a.u (DFT)

Total Energy $=-309.27487338$ a.u (CCSD) 
$\begin{array}{llll}1 & -0.168688 & -0.166976 & -2.492217\end{array}$

$\begin{array}{llll}1 & -1.474330 & -0.666323 & -1.433017\end{array}$

$\begin{array}{llll}8 & 0.745329 & 1.405166 & 0.000000\end{array}$

C. $C_{s}$

\begin{tabular}{lrcc}
\hline Atomic & \multicolumn{3}{c}{ Coordinates (Angstroms) } \\
Number & $X$ & $Y$ & $Z$ \\
& & & \\
\hline 6 & 0.293823 & 1.004585 & 0.735913 \\
6 & 0.293823 & -1.519715 & 0.778646 \\
6 & -0.286837 & -0.229707 & -1.402870 \\
6 & 0.293823 & -1.519715 & -0.778646 \\
6 & 0.293823 & 1.004585 & -0.735913 \\
6 & -0.286837 & -0.229707 & 1.402870 \\
1 & -0.095343 & -0.219420 & -2.479093 \\
1 & 1.064732 & 1.555500 & -1.270568 \\
1 & 1.064732 & 1.555500 & 1.270568 \\
1 & 1.319442 & -1.647133 & 1.138111 \\
1 & 1.319442 & -1.647133 & -1.138111 \\
1 & -0.095343 & -0.219420 & 2.479093 \\
8 & -0.614126 & 1.839692 & 0.000000 \\
1 & -0.265034 & -2.379314 & 1.158451 \\
1 & -0.265034 & -2.379314 & -1.158451 \\
1 & -1.372146 & -0.199375 & 1.273939 \\
1 & -1.372146 & -0.199375 & -1.273939
\end{tabular}

D. $C_{2}$

\begin{tabular}{lrcc}
\hline \multicolumn{3}{l}{$\begin{array}{l}\text { Atomic } \\
\text { Number }\end{array}$} & \multicolumn{3}{l}{ Coordinates (Angstroms) } \\
& & $Y$ & $Z$ \\
\hline 1 & 1.122891 & 2.328253 & -0.201837 \\
6 & 0.999611 & 1.241672 & -0.168012 \\
6 & 0.000000 & -0.724901 & 0.839725 \\
6 & -0.268645 & -0.740313 & -1.475653 \\
6 & -0.999611 & -1.241672 & -0.168012 \\
6 & 0.268645 & 0.740313 & -1.475653 \\
6 & 0.000000 & 0.724901 & 0.839725 \\
1 & 0.977470 & -1.186487 & 0.697282 \\
1 & 0.578986 & -1.412593 & -1.647578 \\
1 & -1.984557 & -0.783615 & -0.054142 \\
1 & -0.578986 & 1.412593 & -1.647578 \\
1 & -0.977470 & 1.186487 & 0.697282 \\
1 & 1.984557 & 0.783615 & -0.054142 \\
1 & -0.929878 & -0.860563 & -2.338627 \\
1 & -1.122891 & -2.328253 & -0.201837 \\
1 & 0.929878 & 0.860563 & -2.338627
\end{tabular}



Total Energy $=-309.921493 a \cdot u($ DFT)

Total Energy $=-309.270897 a . u(C C S D)$

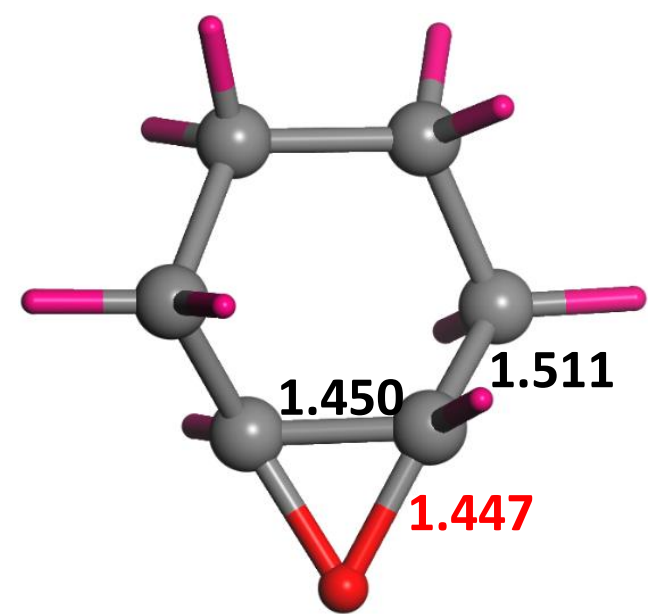

Total Energy = -309.868104a.u (DFT)

Total Energy $=-309.216622 a \cdot u(C C S D)$ 
E. $C_{2 h}$

\begin{tabular}{lrrr}
\hline Atomic & \multicolumn{3}{c}{ Coordinates (Angstroms) } \\
Number & $X$ & $Y$ & $Z$ \\
& & & \\
\hline 1 & 0.404152 & 2.148150 & 1.244201 \\
6 & 0.000000 & 1.273988 & 0.736238 \\
6 & 0.000000 & 0.000000 & -1.550027 \\
6 & 0.000000 & -1.273988 & 0.736238 \\
6 & 0.000000 & -1.273988 & -0.736238 \\
6 & 0.000000 & 0.000000 & 1.550027 \\
6 & 0.000000 & 1.273988 & -0.736238 \\
1 & -0.879963 & 0.013118 & -2.202745 \\
1 & -0.404152 & -2.148150 & 1.244201 \\
1 & 0.879963 & -0.013118 & 2.202745 \\
1 & 0.404152 & 2.148150 & -1.244201 \\
1 & 0.879963 & -0.013118 & -2.202745 \\
1 & -0.404152 & -2.148150 & -1.244201 \\
1 & -0.879963 & 0.013118 & 2.202745 \\
8 & -1.201618 & 1.541956 & 0.000000 \\
8 & 1.201618 & -1.541956 & 0.000000
\end{tabular}

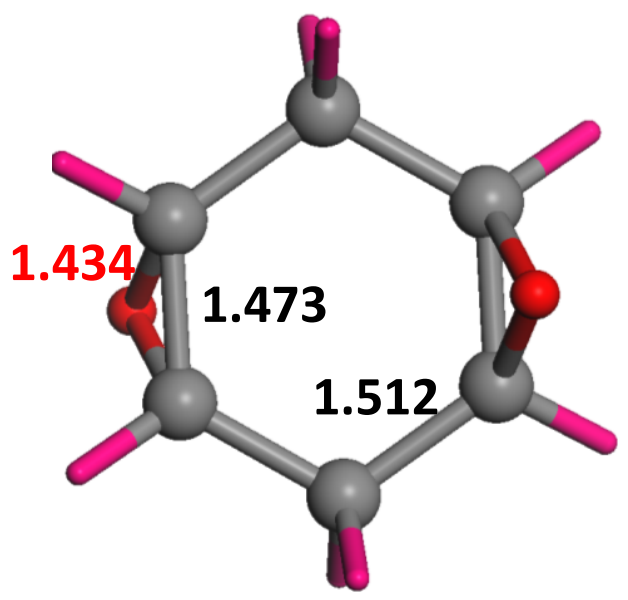

Total Energy = -383.92172223 a.u (DFT)

Total Energy $=-383.16048469$ a.u (CCSD)

F. $C_{2}$

\begin{tabular}{lrrr}
\hline Atomic & \multicolumn{3}{c}{ Coordinates (Angstroms) } \\
Number & $X$ & $Y$ & $Z$ \\
& & & \\
\hline 6 & -0.201709 & 1.449049 & 0.123726 \\
6 & -0.180486 & -0.745429 & 1.408426 \\
6 & 0.180486 & -0.726202 & -1.152016 \\
6 & 0.201709 & -1.449049 & 0.123726 \\
6 & -0.180486 & 0.726202 & -1.152016 \\
6 & 0.180486 & 0.745429 & 1.408426 \\
1 & 0.300045 & -1.242056 & 2.257478 \\
1 & -0.300045 & 1.242056 & 2.257478 \\
1 & -1.260709 & -0.866773 & 1.535708 \\
1 & 0.012734 & 1.276080 & -2.070283 \\
1 & 1.260709 & 0.866773 & 1.535708 \\
1 & -0.011129 & 2.520909 & 0.100807 \\
8 & -1.430768 & 1.069699 & -0.513066 \\
1 & 0.011129 & -2.520909 & 0.100807 \\
1 & -0.012734 & -1.276080 & -2.070283 \\
8 & 1.430768 & -1.069699 & -0.513066
\end{tabular}

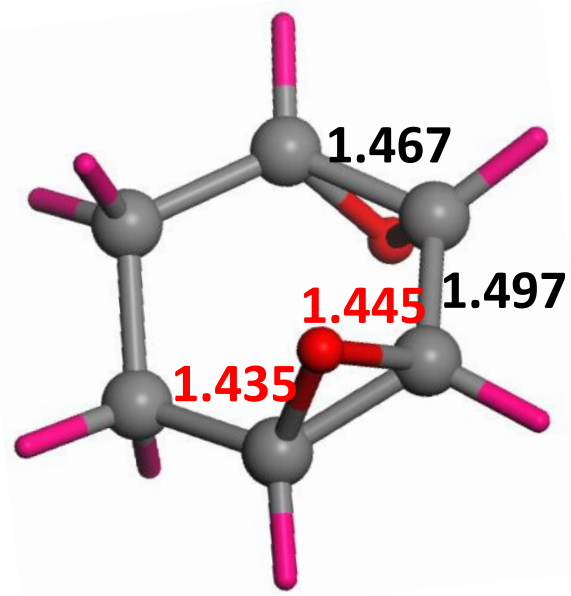

Total Energy $=-383.92011601$ a.u (DFT)

Total Energy = -383.15845594 a.u (CCSD) 
G. $C_{2 v}$

\begin{tabular}{lrrr}
\hline Atomic & \multicolumn{3}{c}{ Coordinates (Angstroms) } \\
Number & $X$ & $Y$ & $Z$ \\
& & & \\
\hline 1 & 2.168459 & 1.248021 & -0.625996 \\
6 & 1.278751 & 0.735488 & -0.261106 \\
6 & -1.278751 & 0.735488 & -0.261106 \\
6 & 0.000000 & -1.537050 & -0.373567 \\
6 & -1.278751 & -0.735488 & -0.261106 \\
6 & 1.278751 & -0.735488 & -0.261106 \\
6 & 0.000000 & 1.537050 & -0.373567 \\
1 & 0.000000 & -2.326691 & 0.387092 \\
1 & -2.168459 & -1.248021 & -0.625996 \\
1 & 2.168459 & -1.248021 & -0.625996 \\
1 & 0.000000 & 2.041497 & -1.347881 \\
1 & -2.168459 & 1.248021 & -0.625996 \\
1 & 0.000000 & -2.041497 & -1.347881 \\
1 & 0.000000 & 2.326691 & 0.387092 \\
8 & -1.497330 & 0.000000 & 0.948432 \\
8 & 1.497330 & 0.000000 & 0.948432
\end{tabular}

H. $C_{1}$

\begin{tabular}{lrrc}
\hline Atomic & \multicolumn{3}{c}{ Coordinates (Angstroms) } \\
Number & $X$ & $Y$ & $Z$ \\
& & & \\
\hline 1 & -0.703361 & -2.216551 & -0.766566 \\
6 & -0.454903 & -1.236244 & -0.365883 \\
6 & 1.314650 & 0.615634 & -0.267159 \\
6 & -1.154105 & 1.249647 & -0.284397 \\
6 & 0.241859 & 1.527430 & 0.309762 \\
6 & -1.517016 & -0.218563 & -0.240090 \\
6 & 0.964181 & -0.779486 & -0.583160 \\
1 & -1.905548 & 1.829071 & 0.261431 \\
1 & 0.507748 & 2.573046 & 0.135757 \\
1 & -2.523130 & -0.477943 & -0.565395 \\
1 & 1.491104 & -1.299895 & -1.381589 \\
1 & 2.116480 & 1.086713 & -0.831579 \\
1 & -1.201525 & 1.580088 & -1.329784 \\
8 & -1.056913 & -0.951697 & 0.900125 \\
1 & 0.212840 & 1.369868 & 1.389599 \\
8 & 1.761587 & -0.472666 & 0.559086
\end{tabular}
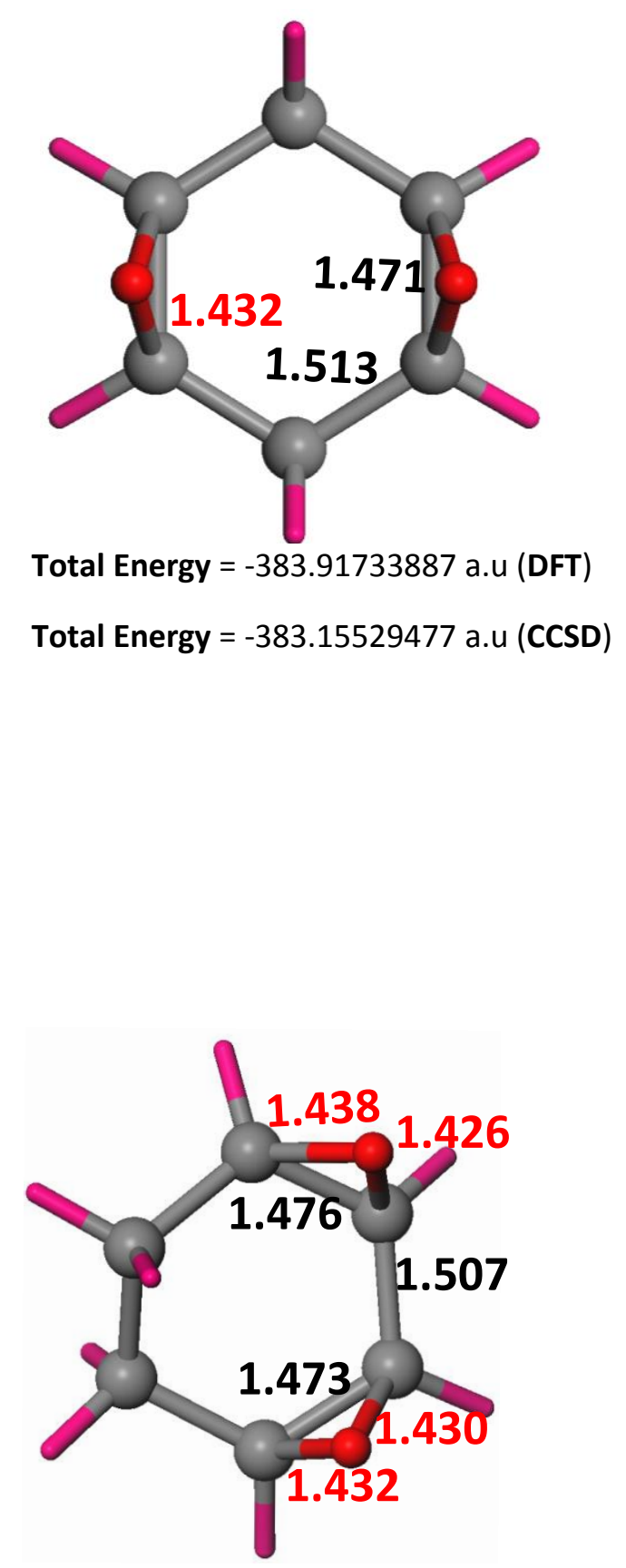

Total Energy $=-383.91324978$ a.u (DFT)

Total Energy $=-383.15119592$ a.u (CCSD) 
I. $C_{s}$

\begin{tabular}{lccc}
\hline Atomic & \multicolumn{3}{c}{ Coordinates (Angstroms) } \\
Number & $X$ & $Y$ & $Z$ \\
\hline 6 & -0.140249 & 0.065564 & 1.493885 \\
6 & 0.522784 & 1.170313 & -0.734039 \\
6 & -0.724640 & -1.062321 & -0.755207 \\
6 & -0.140249 & 0.065564 & -1.493885 \\
6 & -0.724640 & -1.062321 & 0.755207 \\
6 & 0.522784 & 1.170313 & 0.734039 \\
1 & -1.547928 & -1.607813 & -1.210957 \\
1 & 1.341405 & 1.672057 & 1.245800 \\
1 & -0.542205 & 0.328423 & 2.470308 \\
1 & 1.341405 & 1.672057 & -1.245800 \\
1 & -0.542205 & 0.328423 & -2.470308 \\
1 & -1.547928 & -1.607813 & 1.210957 \\
8 & -0.345230 & 2.052138 & 0.000000 \\
8 & 0.522784 & -1.205320 & 1.436469 \\
8 & 0.522784 & -1.205320 & -1.436469
\end{tabular}

J. $C_{3 v}$

\begin{tabular}{lrcc}
\hline Atomic & \multicolumn{3}{c}{ Coordinates (Angstroms) } \\
Number & $X$ & $Y$ & $Z$ \\
& & & \\
\hline 1 & 1.261118 & 2.128316 & -0.852535 \\
6 & 0.742613 & 1.296179 & -0.380228 \\
6 & -1.493830 & -0.004968 & -0.380228 \\
6 & 0.751217 & -1.291211 & -0.380228 \\
6 & -0.751217 & -1.291211 & -0.380228 \\
6 & 1.493830 & -0.004968 & -0.380228 \\
6 & -0.742613 & 1.296179 & -0.380228 \\
1 & -1.212617 & -2.156318 & -0.852535 \\
1 & 2.473735 & 0.028002 & -0.852535 \\
1 & -1.261118 & 2.128316 & -0.852535 \\
1 & -2.473735 & 0.028002 & -0.852535 \\
1 & 1.212617 & -2.156318 & -0.852535 \\
8 & 0.000000 & 1.642334 & 0.783475 \\
8 & -1.422303 & -0.821167 & 0.783475 \\
8 & 1.422303 & -0.821167 & 0.783475
\end{tabular}

K. $C_{1}$

\begin{tabular}{llcc}
\hline Atomic & \multicolumn{3}{c}{ Coordinates (Angstroms) } \\
Number & X & Y & Z \\
& & & \\
\hline 6 & -0.707790 & -1.345845 & -0.256414
\end{tabular}
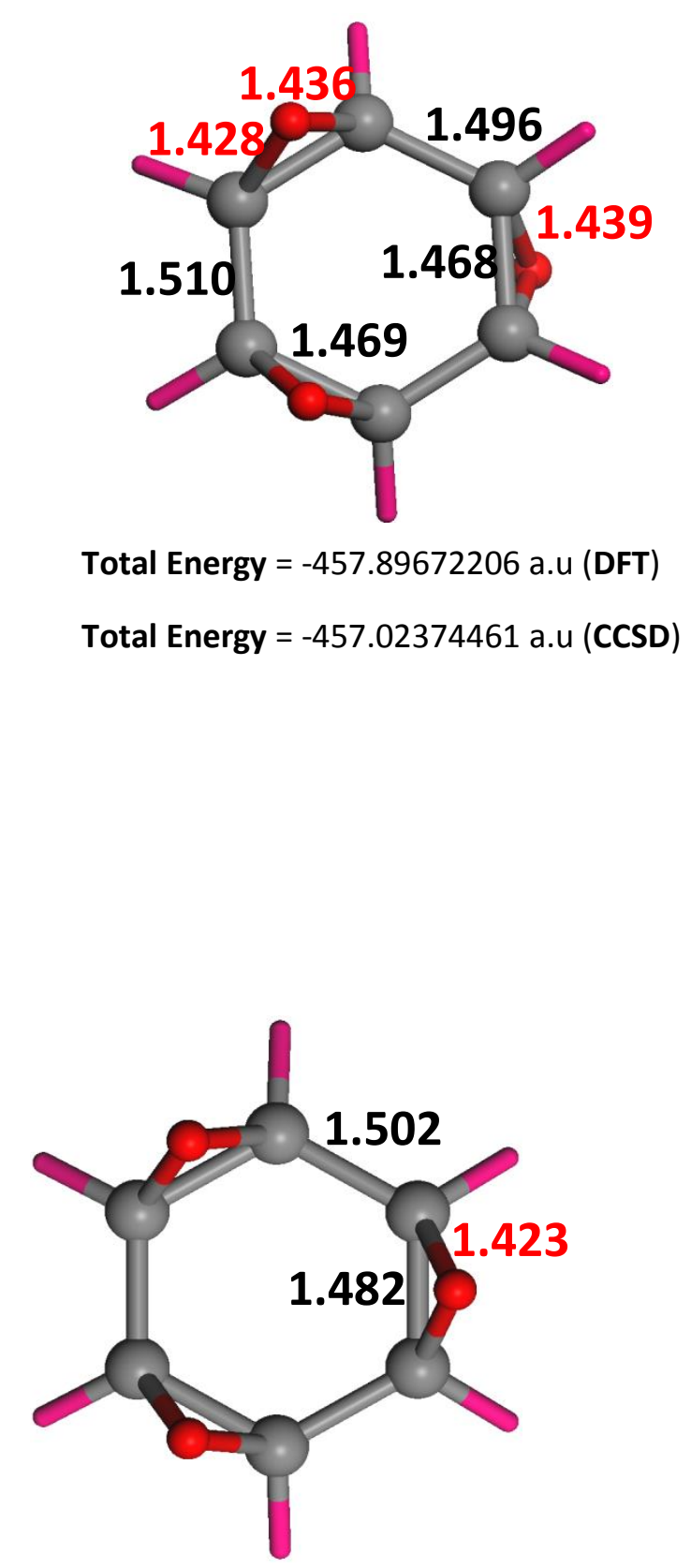

Total Energy $=-457.88926766$ a.u (DFT)

Total Energy $=-457.01494926$ a.u (CCSD) 


$\begin{array}{rrrr}6 & -0.691427 & 1.290785 & -0.345652 \\ 6 & 1.298549 & -0.016944 & -0.496589 \\ 6 & 0.815738 & 1.377817 & -0.234521 \\ 6 & 0.613475 & -0.957470 & 0.381755 \\ 6 & -1.407076 & -0.056421 & -0.558187 \\ 1 & 1.331646 & 2.265221 & -0.589362 \\ 1 & -0.893853 & -2.254581 & -0.821306 \\ 8 & -0.035210 & 1.576902 & 0.889740 \\ 8 & 2.026546 & -1.090225 & 0.135417 \\ 8 & -1.770810 & -0.867493 & 0.567164 \\ 1 & -1.219680 & 2.142129 & -0.766931 \\ 1 & 0.434880 & -0.615326 & 1.394494 \\ 1 & -2.170719 & -0.015107 & -1.332409 \\ 1 & 1.224701 & -0.227344 & -1.565404\end{array}$

L. $C_{2}$

\begin{tabular}{lrrr}
\hline \multirow{2}{*}{$\begin{array}{l}\text { Atomic } \\
\text { Number }\end{array}$} & \multicolumn{3}{c}{ Coordinates (Angstroms) } \\
& & $Y$ & $Z$ \\
\hline 1 & 1.265437 & -0.513590 & -2.011981 \\
6 & 0.511724 & -0.570211 & -1.230203 \\
6 & -0.104655 & -0.723524 & 1.104918 \\
6 & -0.797807 & 1.315205 & 0.033871 \\
6 & 0.104655 & 0.723524 & 1.104918 \\
6 & -0.511724 & 0.570211 & -1.230203 \\
6 & 0.797807 & -1.315205 & 0.033871 \\
1 & -1.128274 & -1.052217 & 0.938987 \\
1 & -1.767117 & 1.766668 & 0.223412 \\
1 & 1.128274 & 1.052217 & 0.938987 \\
1 & 1.767117 & -1.766668 & 0.223412 \\
1 & -1.265437 & 0.513590 & -2.011981 \\
8 & 0.000000 & -1.899295 & -0.996093 \\
8 & 0.000000 & 1.899295 & -0.996093 \\
8 & 0.000000 & 0.000000 & 2.341702
\end{tabular}
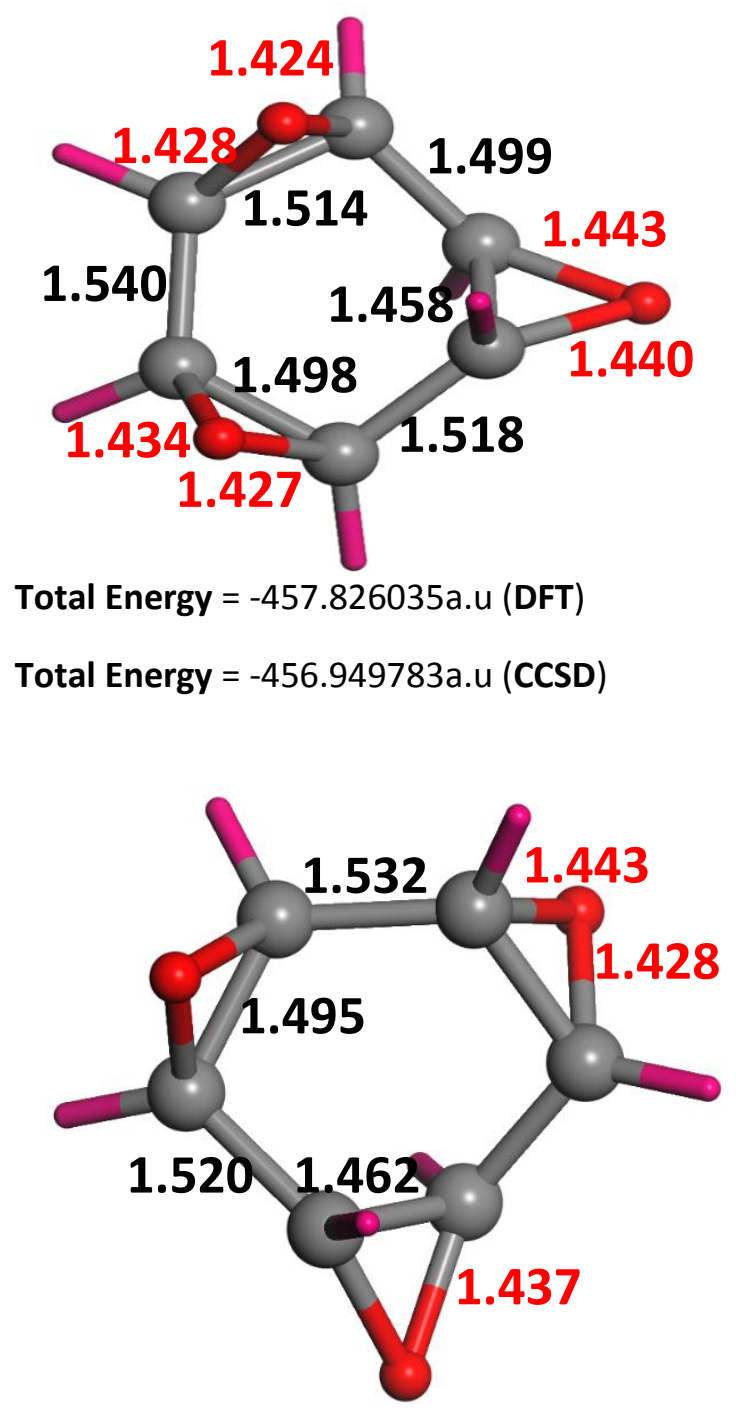

Total Energy $=-457.823308 a \cdot u(D F T)$

Total Energy $=-456.946875 a \cdot u(C C S D)$ 
S2. Construction of the isomers of $\mathrm{C}_{2} \mathrm{O}$. The schematic enumeration of $\mathrm{C}_{2} \mathrm{O}$ isomers by bringing 2-4 zig-zag chains having epoxides together collaterally. Epoxides bridges above and below are given in distinct shades of pink. There are four distinct zig-zag chains (a) all cis (b) alternating cis and trans (c) two consecutive cis and trans (d) non conjugated (separated by more than two $\mathrm{C}-\mathrm{C}$ bonds). These chains can be connected to in such a way that they can produce either the intra (First Column) or intra (Second column) epoxide interactions. Systems having more than two consecutive epoxides in cis will violate their equivalence. Similarly, merging of more than four zig-zag chains also will violate their equivalence. Even within these constraints, non-equivalent isomers are generated but identified and ignored. Redundant structures generated are given with the same symbol.

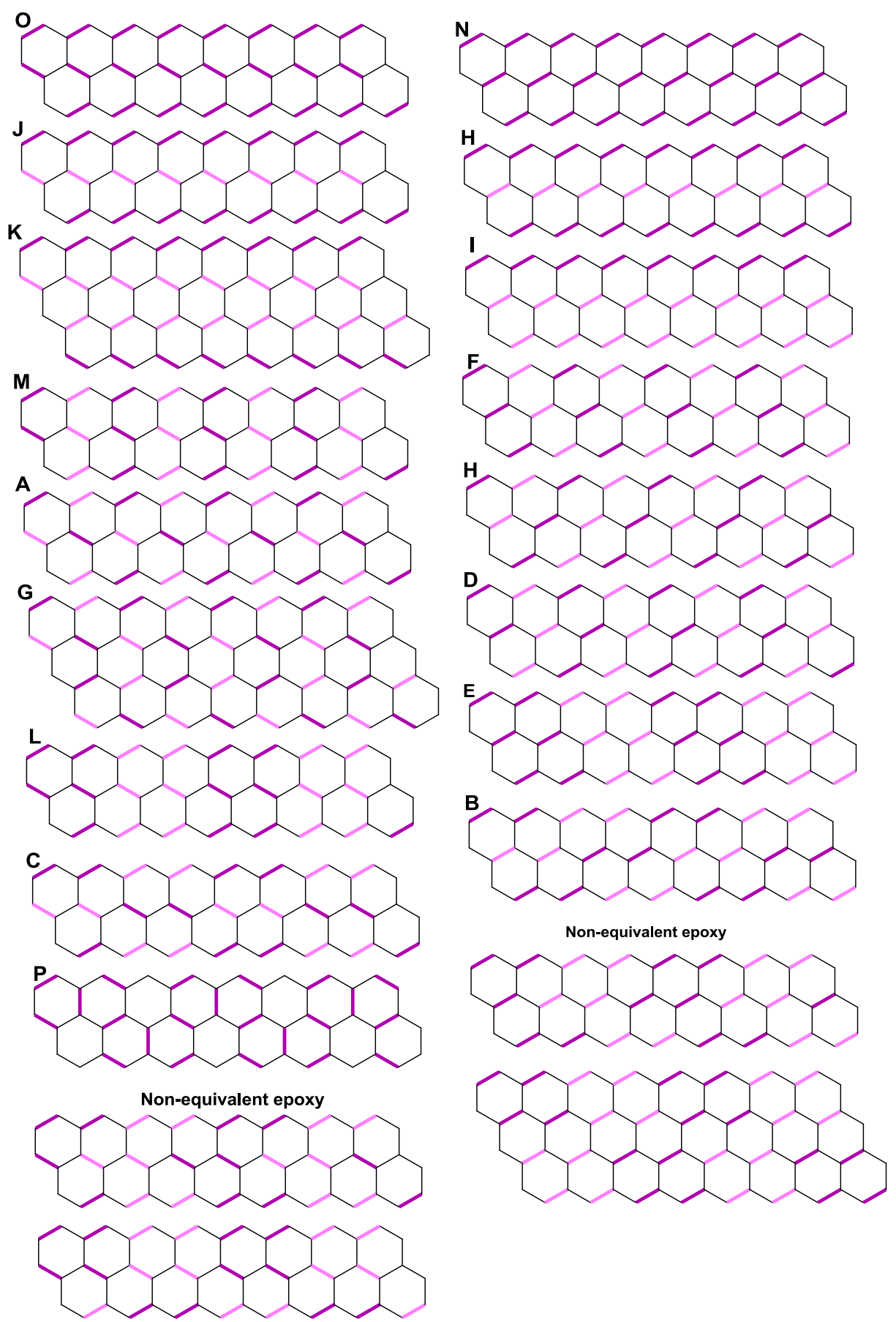




\section{S3. Phonon dispersion of GO nanosheets.}

Dispersion corrected GGA, norm-conserving pseudo-potentials,

Linear response method using Koelling-Harmon relativistic treatment

Plane-wave cut-off $830 \mathrm{eV}$; K-mesh: Monkhorst-Pack ${ }^{49}$ with 0.07 Å separation.
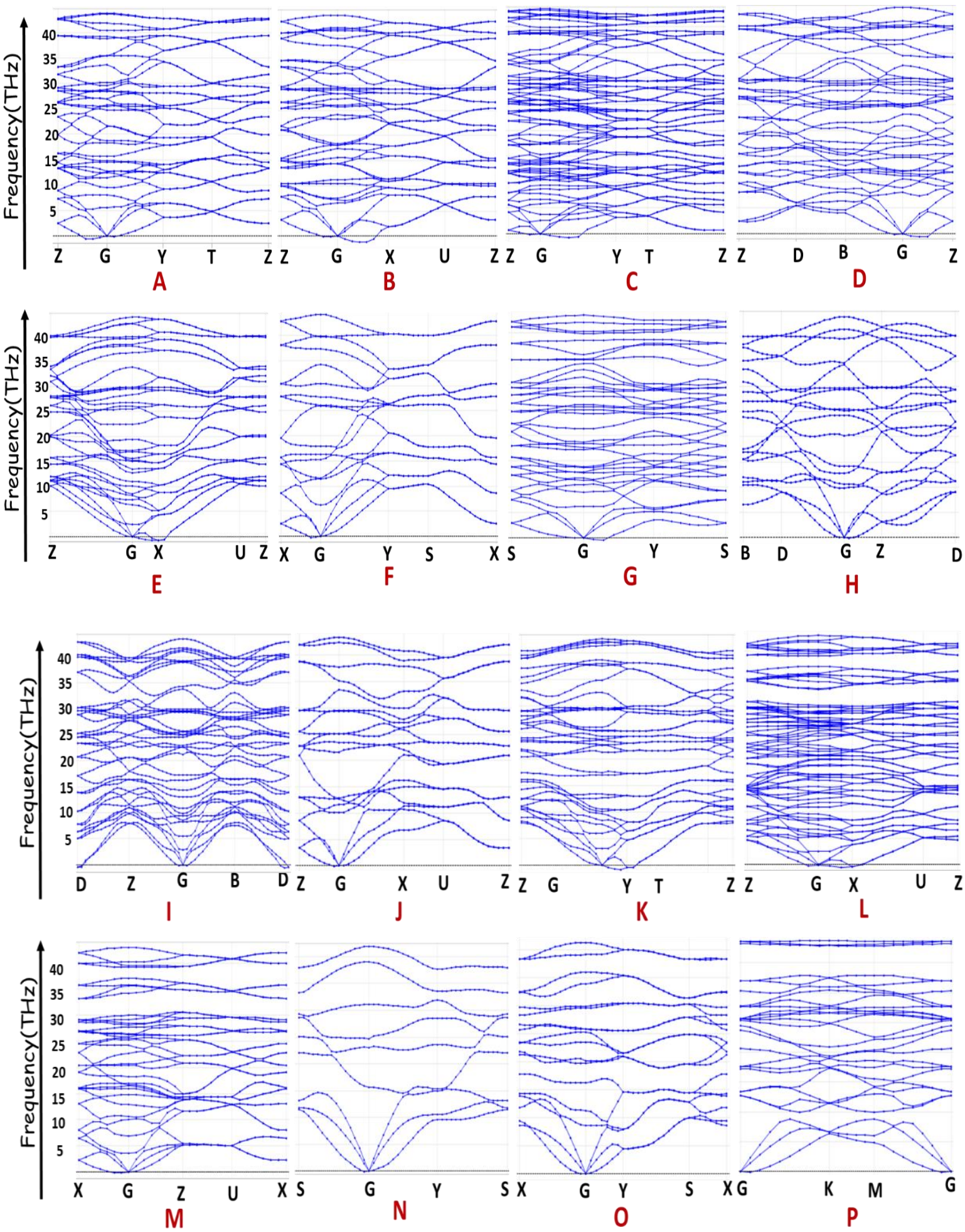


\section{S4. Band structures of GO nanosheets}

Dispersion corrected GGA, ultra-soft pseudo-potentials,

Plane-wave cut-off $600 \mathrm{eV}$; K-point separation $0.04 \AA$ Å.
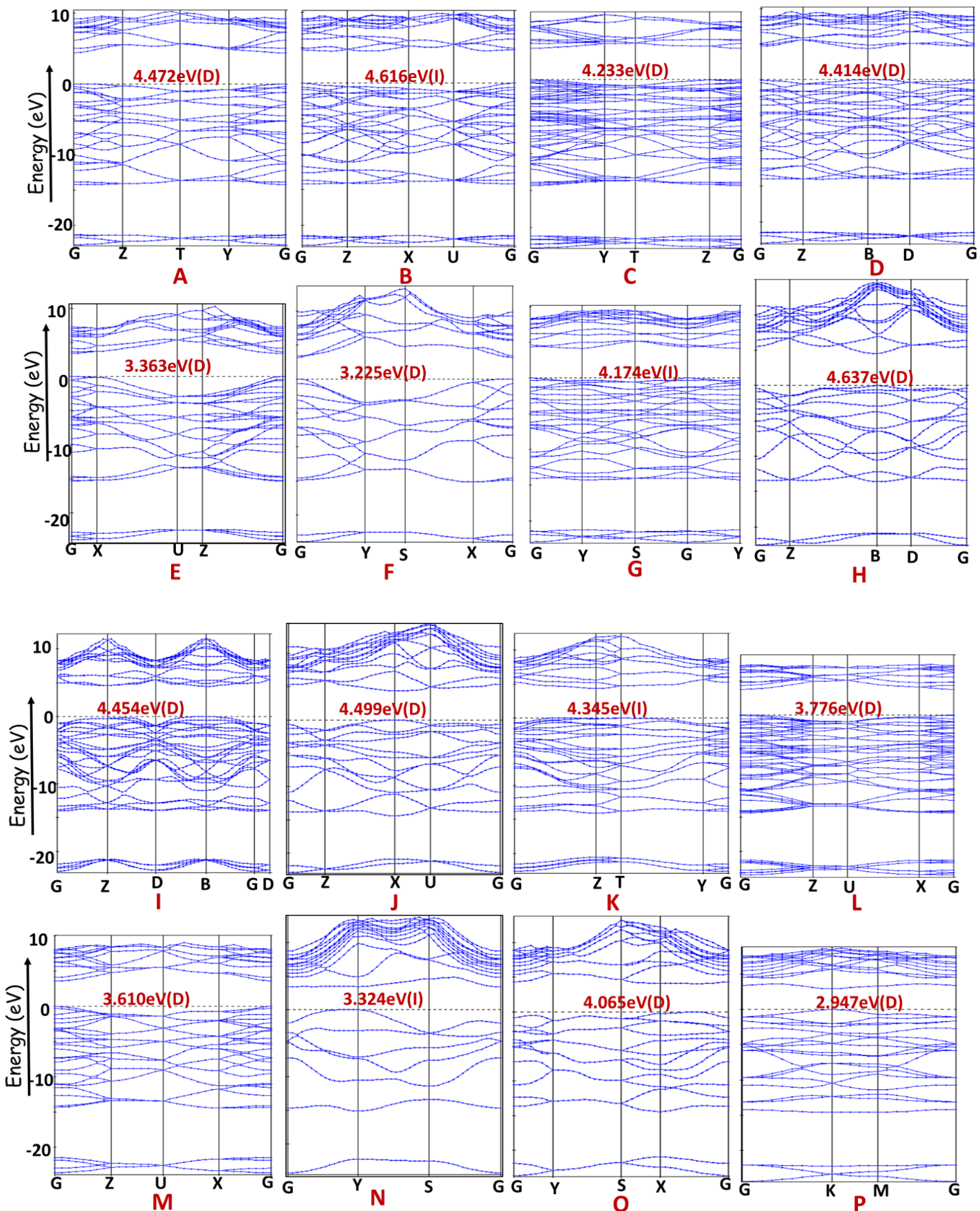
S5. Density of State of GO nanosheets.

Dispersion corrected GGA, ultra-soft pseudo-potentials

Plane-wave cut-off $600 \mathrm{eV}$; K-point Mesh separation: $0.04 \AA$
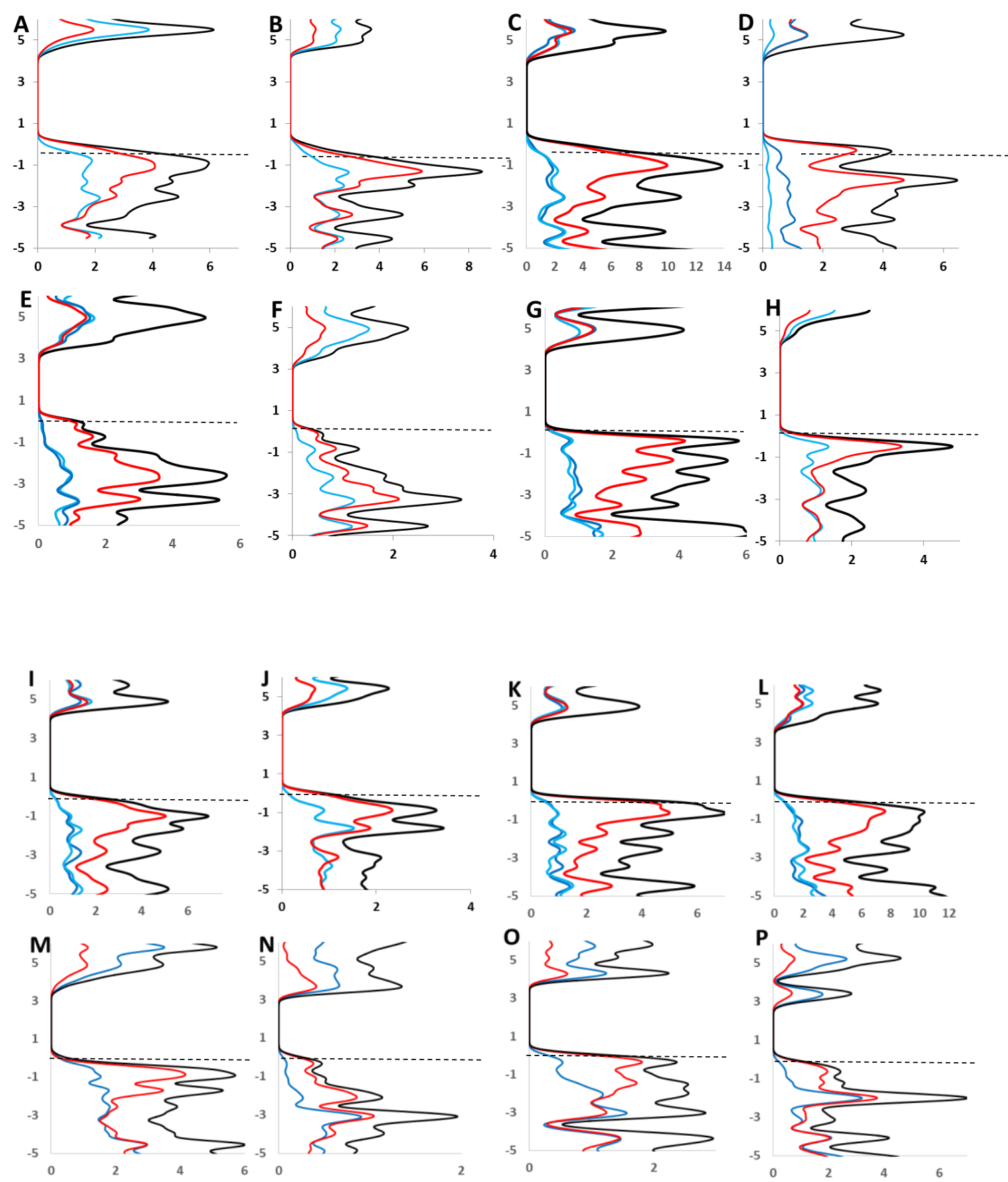

PDOS of $\mathrm{C} 1$
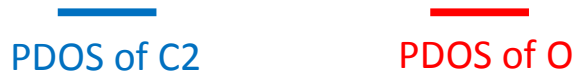

Total DOS 
S6. COOP (Crystal orbital overlap population) plots of GO nanosheets.

Geometry : Dispersion corrected GGA/ultrasoft pseudopotentials COOP curves from YAeHMOP based on extended Huckel theory

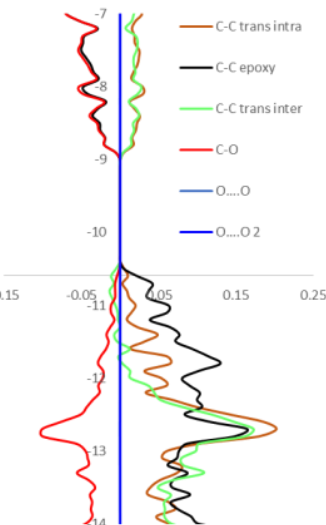

A



E

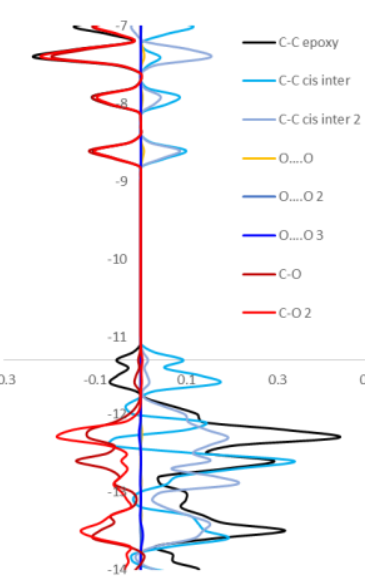

I



M

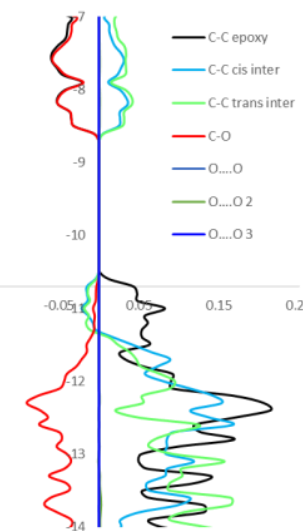

B



$\mathbf{F}$

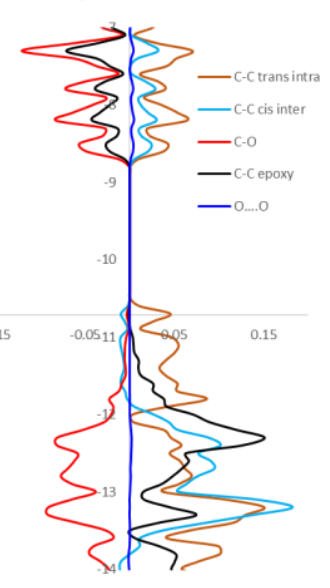

J



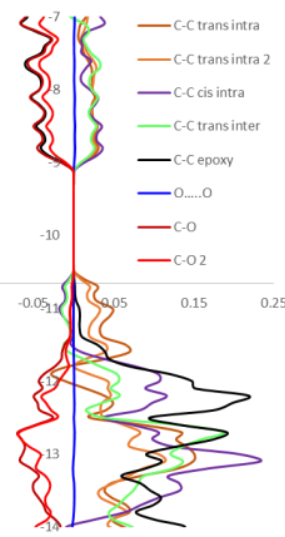

C

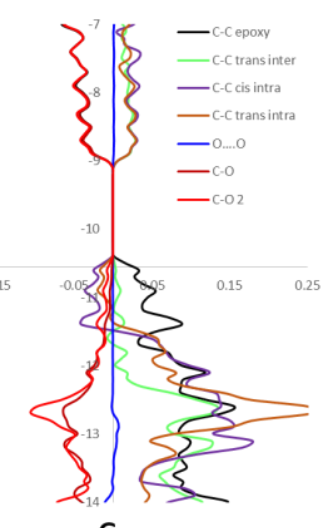

G

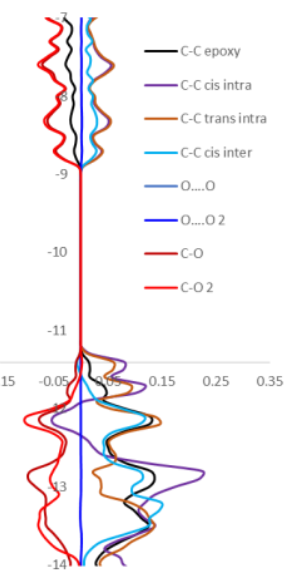

K

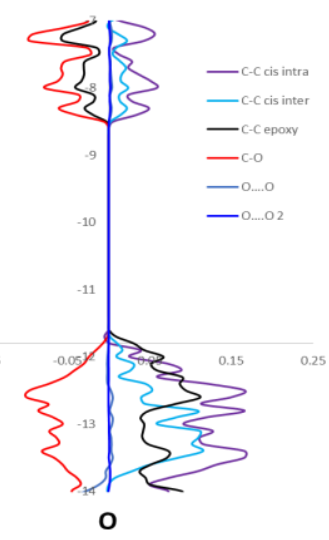

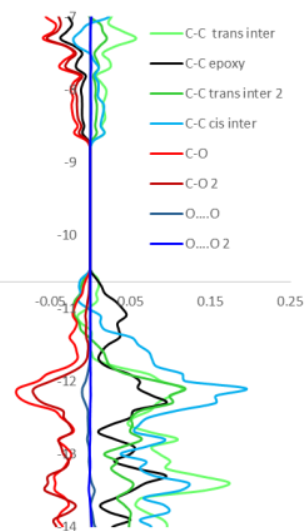

D



$\mathrm{H}$

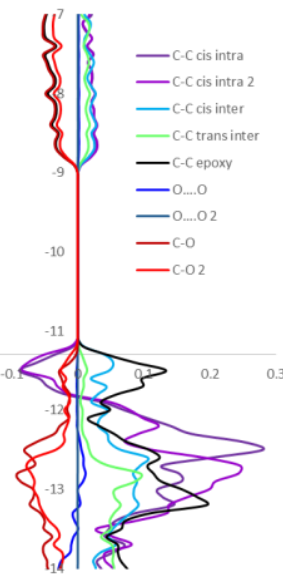

L

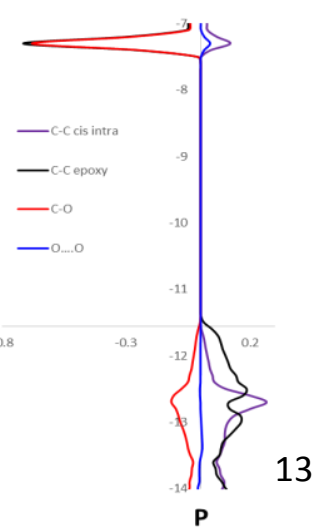


S7. Epoxide dimers with coordinates.

Geometry Optimization:

Method: B3LYP

Basis Set: 6-311G(d,p)

Single Point Calculations:

Method: CCSD

Basis Set: aug-cc-pVTZ

A. $\mathrm{SR}, C_{i}$

\begin{tabular}{lrrr}
\hline \multirow{2}{*}{$\begin{array}{l}\text { Atomic } \\
\text { Number }\end{array}$ X Coordinates (Angstroms) } \\
& & & \\
\hline 1 & 0.915418 & 1.922973 & -1.127016 \\
6 & 0.407881 & 1.911716 & -0.165081 \\
1 & 0.677299 & 2.713694 & 0.517404 \\
6 & -0.085068 & 0.639236 & 0.375736 \\
1 & -0.168012 & 0.530273 & 1.456078 \\
6 & 0.085068 & -0.639236 & -0.375736 \\
1 & 0.168012 & -0.530273 & -1.456078 \\
6 & -0.407881 & -1.911716 & 0.165081 \\
1 & -0.677299 & -2.713694 & -0.517404 \\
1 & -0.915418 & -1.922973 & 1.127016 \\
8 & 0.986507 & -1.585476 & 0.211533 \\
8 & -0.986507 & 1.585476 & -0.211533
\end{tabular}

$8 \quad-1.706808 \quad-0.770798 \quad 0.077917$

B. RS, Cs

\begin{tabular}{|c|c|c|c|}
\hline \multirow{2}{*}{\multicolumn{2}{|c|}{$\begin{array}{l}\text { Atomic } \\
\text { Number }\end{array}$}} & \multicolumn{2}{|c|}{ Coordinates (Angstroms) } \\
\hline & & Y & Z \\
\hline 1 & -0.840099 & 0.574790 & 2.589391 \\
\hline 6 & -0.724475 & 0.135188 & 1.600568 \\
\hline 1 & -1.643562 & -0.216844 & 1.139677 \\
\hline 6 & 0.408864 & 0.545950 & 0.756250 \\
\hline 1 & 1.106421 & 1.266162 & 1.182175 \\
\hline 6 & 0.408864 & 0.545950 & -0.756250 \\
\hline 1 & 1.106421 & 1.266162 & -1.182175 \\
\hline 6 & -0.724475 & 0.135188 & -1.600568 \\
\hline 1 & -1.643562 & -0.216844 & -1.139677 \\
\hline 1 & -0.840099 & 0.574790 & -2.589391 \\
\hline 8 & 0.408864 & -0.713867 & 1.427493 \\
\hline
\end{tabular}
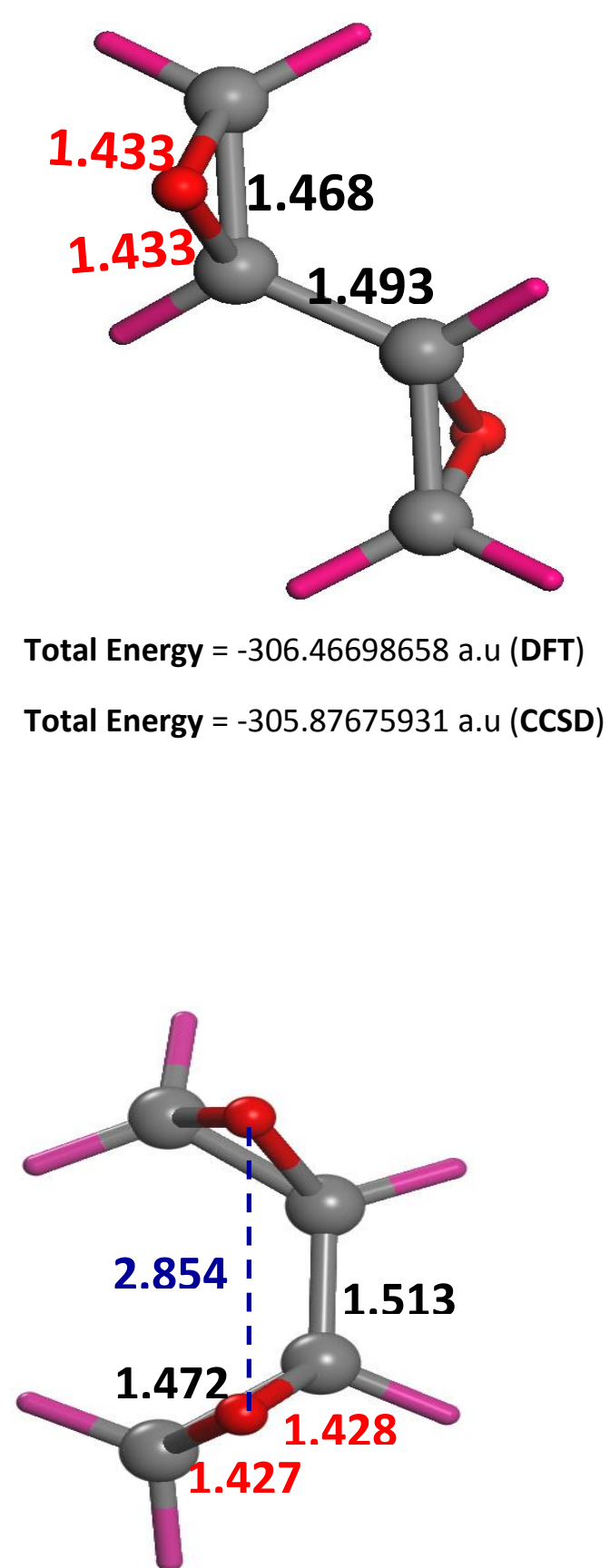

Total Energy = -306.45752204 a.u (DFT)

Total Energy = -305.86752410 a.u (CCSD) 
C. $\mathrm{RR}, \mathrm{C}_{2}$

\begin{tabular}{lrrr}
\hline \multirow{2}{*}{$\begin{array}{l}\text { Atomic } \\
\text { Number }\end{array}$ X } & \multicolumn{3}{l}{$Y$} \\
& & & \\
\hline 6 & -0.005230 & 1.859383 & 0.650034 \\
6 & -0.438373 & 0.601670 & 0.025440 \\
6 & 0.438373 & -0.601670 & 0.025440 \\
6 & 0.005230 & -1.859383 & 0.650034 \\
8 & 0.005230 & 1.708156 & -0.770952 \\
1 & 0.974826 & 1.917180 & 1.119572 \\
1 & -0.752469 & 2.543213 & 1.045645 \\
1 & -1.503698 & 0.390280 & -0.050442 \\
1 & 1.503698 & -0.390280 & -0.050442 \\
1 & -0.974826 & -1.917180 & 1.119572 \\
1 & 0.752469 & -2.543213 & 1.045645 \\
8 & -0.005230 & -1.708156 & -0.770952
\end{tabular}

D. RS, $C_{1}$

\begin{tabular}{lrrc}
\hline \multirow{3}{*}{$\begin{array}{l}\text { Atomic } \\
\text { Number }\end{array}$ X } & \multicolumn{3}{l}{ Coordinates (Angstroms) } \\
& & & \\
\hline 8 & 1.273749 & 0.417124 & -0.801583 \\
6 & 1.827167 & 0.148385 & 0.491107 \\
6 & 0.698579 & -0.636769 & -0.027777 \\
1 & 1.641913 & 0.913032 & 1.241954 \\
1 & 2.825629 & -0.280849 & 0.492187 \\
1 & 0.898423 & -1.632649 & -0.420732 \\
6 & -0.689369 & -0.434506 & 0.491345 \\
6 & -1.436241 & 0.799107 & 0.223146 \\
8 & -1.733392 & -0.409794 & -0.488420 \\
1 & -0.930652 & -1.014375 & 1.382613 \\
1 & -0.959529 & 1.573893 & -0.368671 \\
1 & -2.199456 & 1.125012 & 0.925740
\end{tabular}

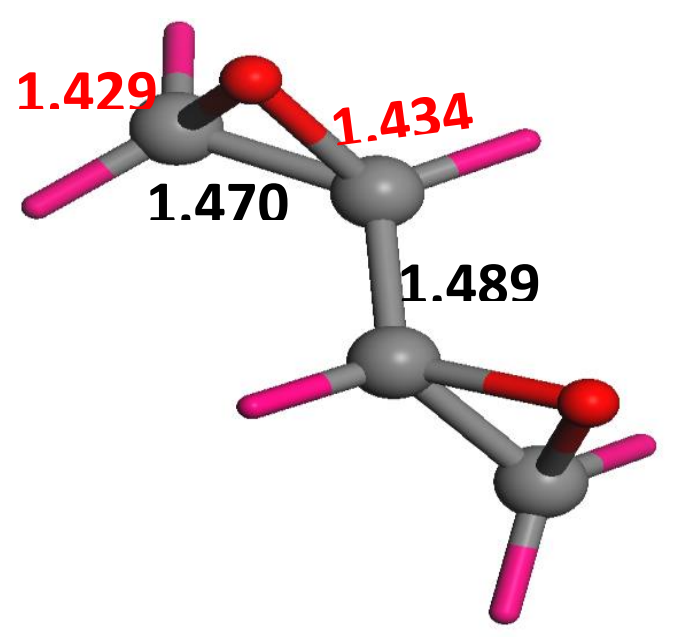

Total Energy $=-306.46697092$ a.u (DFT)

Total Energy = -305.87653305 a.u (CCSD)

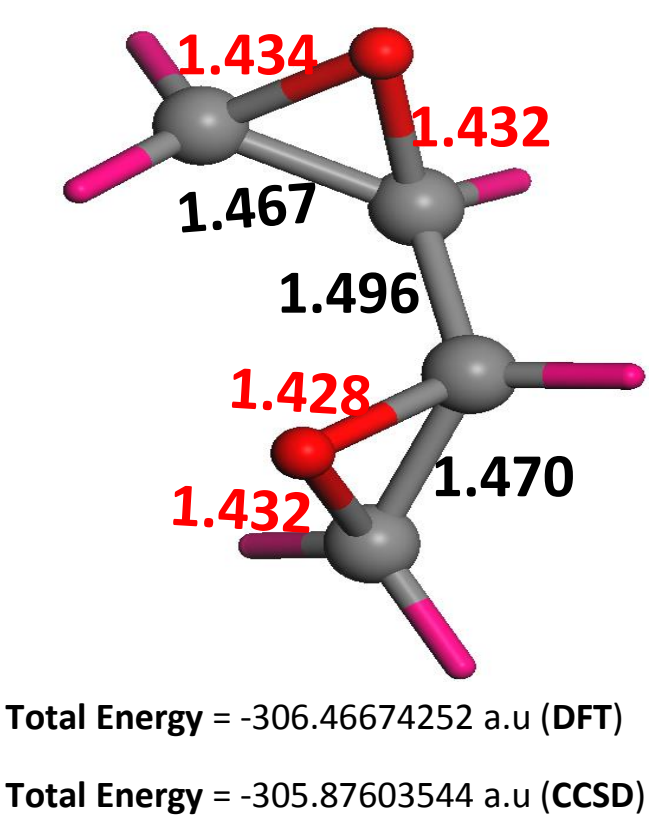


E. $R R, C_{2}$

\begin{tabular}{lll}
\hline Atomic & \multicolumn{3}{c}{ Coordinates } & (Angstroms) \\
Number & $X \quad Y \quad Z$
\end{tabular}

\begin{tabular}{rrrr}
\hline 6 & 0.518462 & -1.509989 & 0.714416 \\
6 & 0.389499 & -0.640174 & -0.461494 \\
1 & 0.049817 & -1.222263 & 1.651446 \\
6 & -0.389499 & 0.640174 & -0.461494 \\
6 & -0.518462 & 1.509989 & 0.714416 \\
8 & 0.389499 & 1.836459 & -0.343822 \\
1 & -1.168913 & 0.685760 & -1.221460 \\
1 & -0.049817 & 1.222263 & 1.651446 \\
1 & -1.384316 & 2.161688 & 0.803054 \\
1 & 1.384316 & -2.161688 & 0.803054 \\
1 & 1.168913 & -0.685760 & -1.221460 \\
8 & -0.389499 & -1.836459 & -0.343822
\end{tabular}

F. $\mathrm{RR}, \boldsymbol{C}_{2}$

\begin{tabular}{lll}
\hline Atomic & \multicolumn{3}{c}{ Coordinates } & (Angstroms) \\
Number & $X$ & $Y$
\end{tabular}

$\begin{array}{lllll}1 & 0.290478 & 2.738487 & -0.178642\end{array}$

$\begin{array}{lllll}6 & 0.157372 & 1.690676 & -0.417028\end{array}$

$\begin{array}{lllll}1 & 0.749839 & 1.340502 & -1.251655\end{array}$

$\begin{array}{llll}6 & -0.157372 & 0.740028 & 0.710893\end{array}$

$\begin{array}{llll}1 & -0.251722 & 1.183701 & 1.698803\end{array}$

$\begin{array}{lllll}8 & -1.149780 & 1.135141 & -0.253963\end{array}$

$\begin{array}{llll}6 & 0.157372 & -0.740028 & 0.710893\end{array}$

$\begin{array}{lllll}1 & 0.251722 & -1.183701 & 1.698803\end{array}$

$\begin{array}{lllll}6 & -0.157372 & -1.690676 & -0.417028\end{array}$

$\begin{array}{lllll}1 & -0.749839 & -1.340502 & -1.251655\end{array}$

$\begin{array}{lllll}1 & -0.290478 & -2.738487 & -0.178642\end{array}$

$\begin{array}{lllll}8 & 1.149780 & -1.135141 & -0.253963\end{array}$
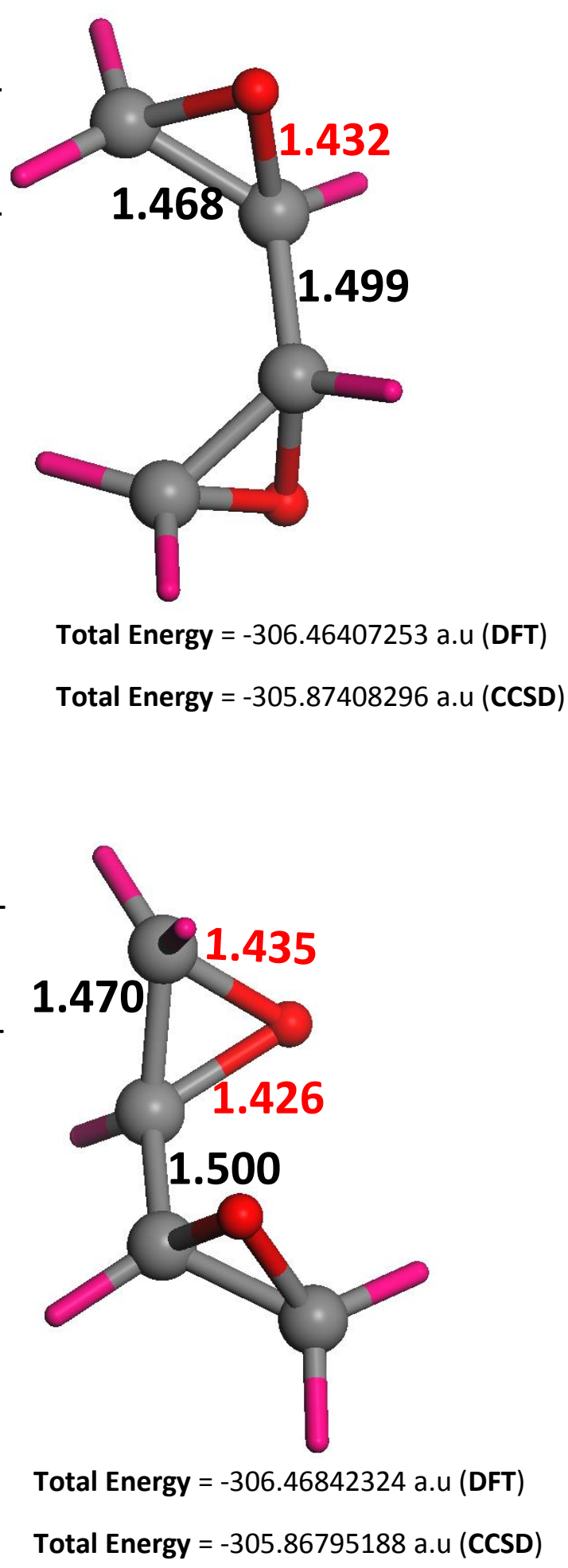
S8. Interaction-correlation diagram of the epoxide trans dimer.
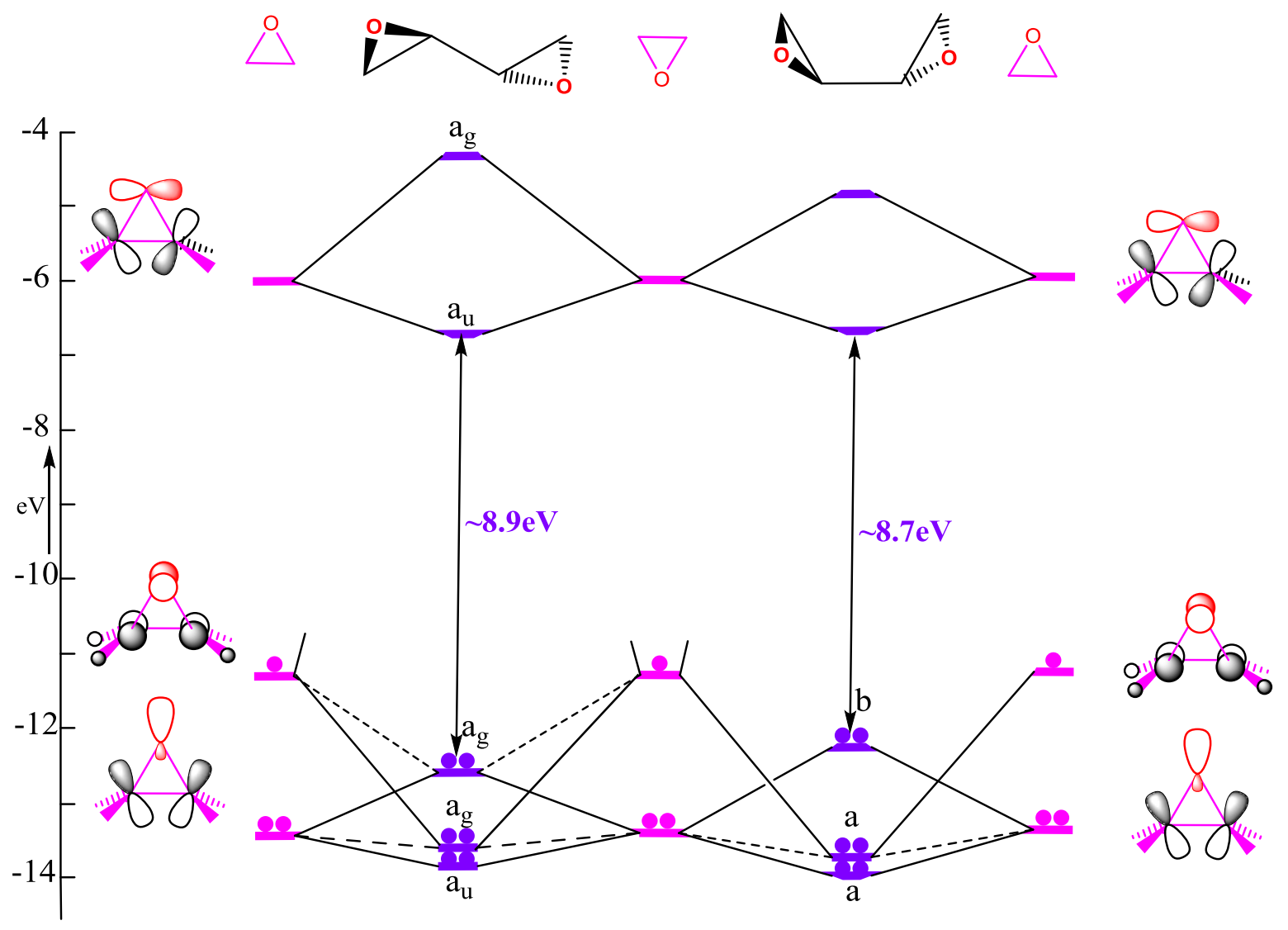
S9. The SALCs of the triangular ring of a sp element

MOs arising from the $s$ and three $p$ orbitals (Radial, tangential and perpendicular). The symmetrical equivalence of $s$ and radial $p$ orbitals justifies $s p$ hybridization among which inward pointing $s p$ hybrid together with tangential $p$ are responsible for ring bonding while the out-ward pointing $s p$ hybrid along with perpendicular $(\pi) p$ orbitals are assumed to engage the substituents.
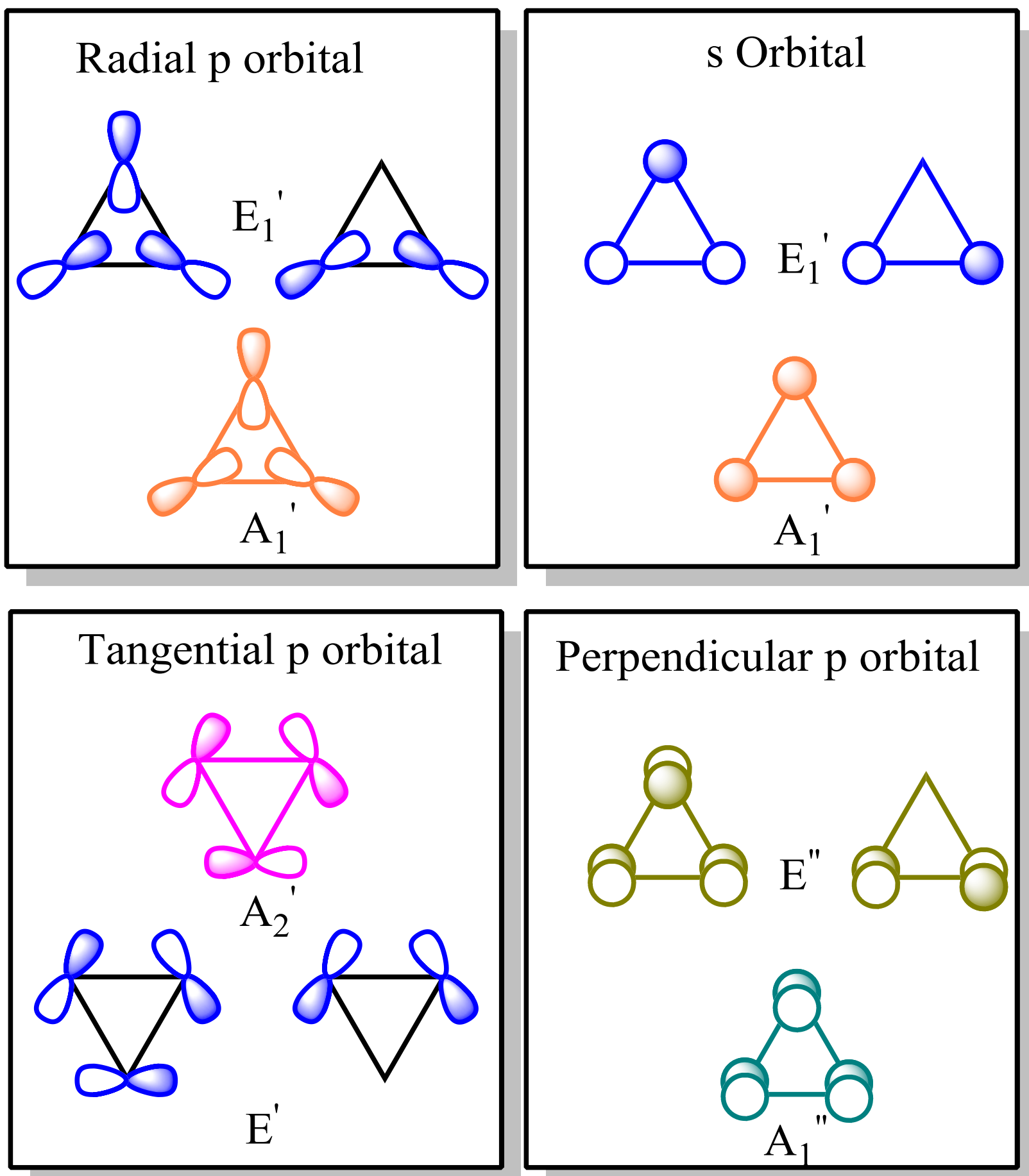

\section{Perpendicular $\mathrm{p}$ orbital}




S10. Topology of GO isomers with coordinates.

Isomer A

Element Fractional coordinates of atoms

u

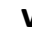

w

\begin{tabular}{llll}
\hline C & 0.583631 & -0.008058 & 0.375391
\end{tabular}

$\begin{array}{llll}\text { C } & -0.083631 & 0.008058 & 0.375391\end{array}$

$\begin{array}{llll}\text { C } & -0.583631 & -0.008058 & 0.124609\end{array}$

C $\quad \begin{array}{llll}1.083631 & 0.008058 & 0.124609\end{array}$

$\begin{array}{llll}\text { C } & -0.583631 & 0.008058 & -0.375391\end{array}$

C $\quad 1.083631-0.008058-0.375391$

$\begin{array}{llll}\text { C } & 0.583631 & 0.008058 & 0.875391\end{array}$

$\begin{array}{llll}\text { C } & -0.083631 & -0.008058 & 0.875391\end{array}$

O $0.500000-0.069979-1.750000$

O $\quad 0.000000 \quad 0.069979-1.750000$

$\begin{array}{llll}0 & -0.500000 & 0.069979 & 1.750000\end{array}$

O $\quad 1.000000-0.069979 \quad 1.750000$

Lattice parameters( $(\AA) \quad$ Cell Angles

$$
\begin{array}{ll}
a=4.403 & \alpha=90.00 \\
b=25.000 & \beta=90.00 \\
c=5.096 & \gamma=90.00
\end{array}
$$

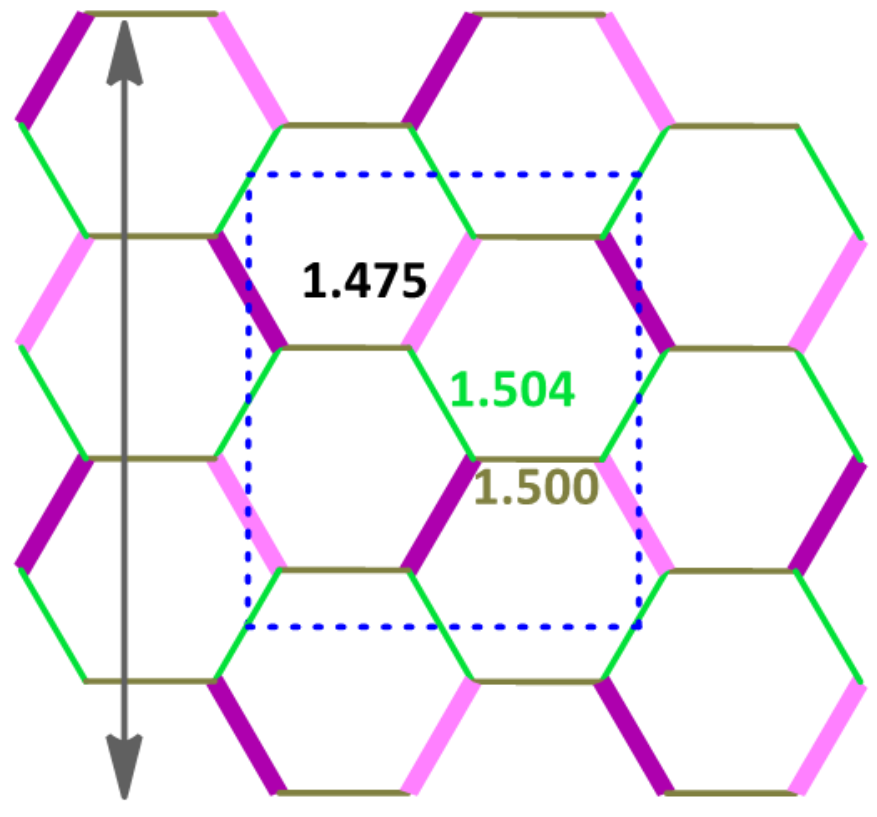

A. $p 2 g g(0.0)$

\section{Isomer B}

Element Fractional coordinates of atoms

u $v \quad w$

$\begin{array}{rrrr}C & 0.014556 & 0.882169 & 0.582766 \\ C & -0.014556 & -0.882169 & 1.082766 \\ C & -0.014556 & 1.382169 & -0.082766 \\ C & 0.014556 & -0.382169 & -0.582766 \\ C & -0.014556 & -0.882169 & -0.582766 \\ C & 0.014556 & 0.882169 & -0.082766 \\ C & 0.014556 & -0.382169 & 1.082766 \\ C & -0.014556 & 1.382169 & 0.582766 \\ O & 0.074415 & 0.939845 & 0.750000 \\ O & -0.074415 & -0.939845 & 1.250000 \\ O & -0.074415 & 1.439845 & -0.250000 \\ O & 0.074415 & -0.439845 & -0.750000\end{array}$

Lattice parameters ( $\AA$ ) Cell Angles

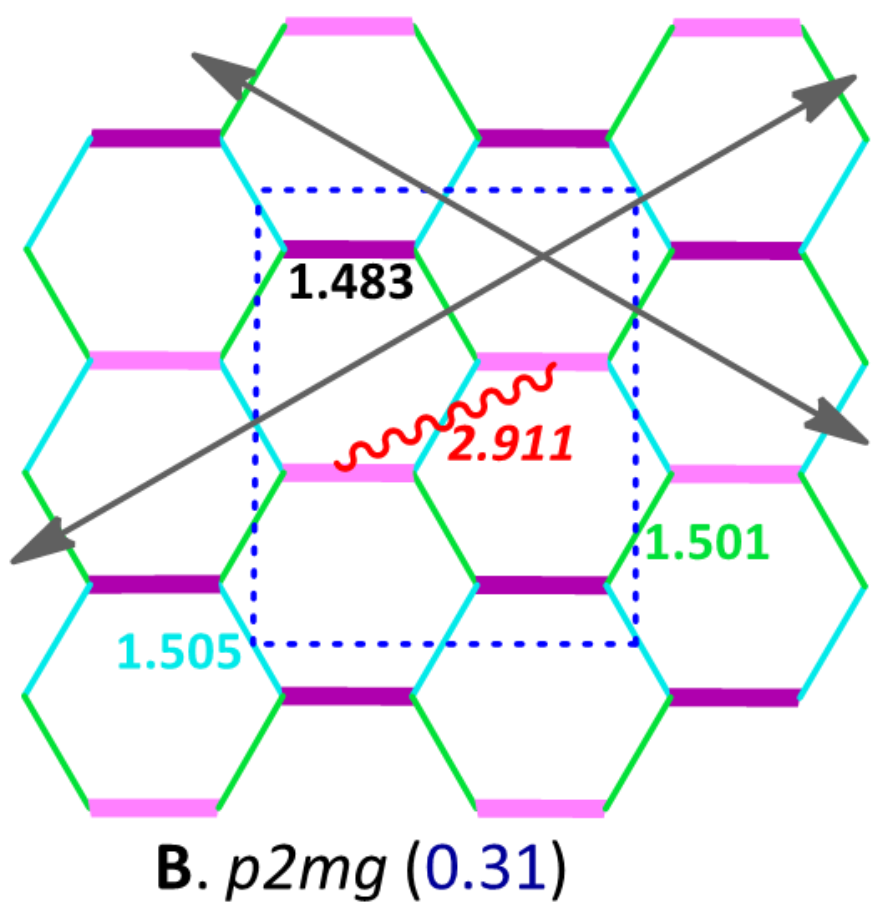

$$
\begin{array}{ll}
a=25.000 & \alpha=90.00 \\
b=4.971 & \beta=90.00 \\
c=4.433 & \gamma=90.00
\end{array}
$$




\section{Isomer C}

Element Fractional coordinates of atoms

\begin{tabular}{|c|c|c|c|}
\hline & u & $\mathbf{v}$ & $\mathbf{w}$ \\
\hline C & 0.585090 & 0.010324 & 0.186221 \\
\hline C & 0.416638 & 0.008521 & 0.061594 \\
\hline C & -0.085090 & -0.010324 & 0.186221 \\
\hline C & 0.083362 & -0.008521 & 0.061594 \\
\hline C & -0.585090 & 0.010324 & 0.313779 \\
\hline C & -0.416638 & 0.008521 & 0.438406 \\
\hline C & 1.085090 & -0.010324 & 0.313779 \\
\hline C & 0.916638 & -0.008521 & 0.438406 \\
\hline C & -0.585090 & -0.010324 & -0.186221 \\
\hline C & -0.416638 & -0.008521 & -0.061594 \\
\hline C & 1.085090 & 0.010324 & -0.186221 \\
\hline C & 0.916638 & 0.008521 & -0.061594 \\
\hline C & 0.585090 & -0.010324 & 0.686221 \\
\hline C & 0.416638 & -0.008521 & 0.561594 \\
\hline C & -0.085090 & 0.010324 & 0.686221 \\
\hline C & 0.083362 & 0.008521 & 0.561594 \\
\hline 0 & 0.509667 & 0.070863 & 0.115954 \\
\hline 0 & -0.009667 & -0.070863 & 0.115954 \\
\hline 0 & -0.509667 & 0.070863 & 0.384046 \\
\hline $\mathrm{O}$ & 1.009667 & -0.070863 & 0.384046 \\
\hline 0 & -0.509667 & -0.070863 & -0.115954 \\
\hline 0 & 1.009667 & 0.070863 & -0.115954 \\
\hline $\mathrm{O}$ & 0.509667 & -0.070863 & 0.615954 \\
\hline 0 & -0.009667 & 0.070863 & 0.615954 \\
\hline
\end{tabular}

Lattice parameters $(\AA \AA) \quad$ Cell Angles

$$
\begin{array}{ll}
a=4.380 & \alpha=90.00 \\
b=25.000 & \beta=90.00 \\
c=10.296 & \gamma=90.00
\end{array}
$$

Isomer D

Element Fractional coordinates of atoms

$\mathbf{u}$

w

$\begin{array}{lrrr}C & 0.333127 & -0.006487 & 0.493628 \\ C & 0.168545 & 0.011082 & 0.243741 \\ C & -0.333127 & -0.006487 & 0.006372 \\ C & -0.168545 & 0.011082 & 0.256259 \\ C & -0.333127 & 0.006487 & -0.493628 \\ C & -0.168545 & -0.011082 & -0.243741 \\ C & 0.333127 & 0.006487 & 0.993628 \\ C & 0.168545 & -0.011082 & 0.743741 \\ O & 0.253099 & -0.070007 & -1.400891 \\ O & -0.253099 & -0.070007 & 1.900891 \\ O & -0.253099 & 0.070007 & 1.400891 \\ O & 0.253099 & 0.070007 & -0.900891\end{array}$

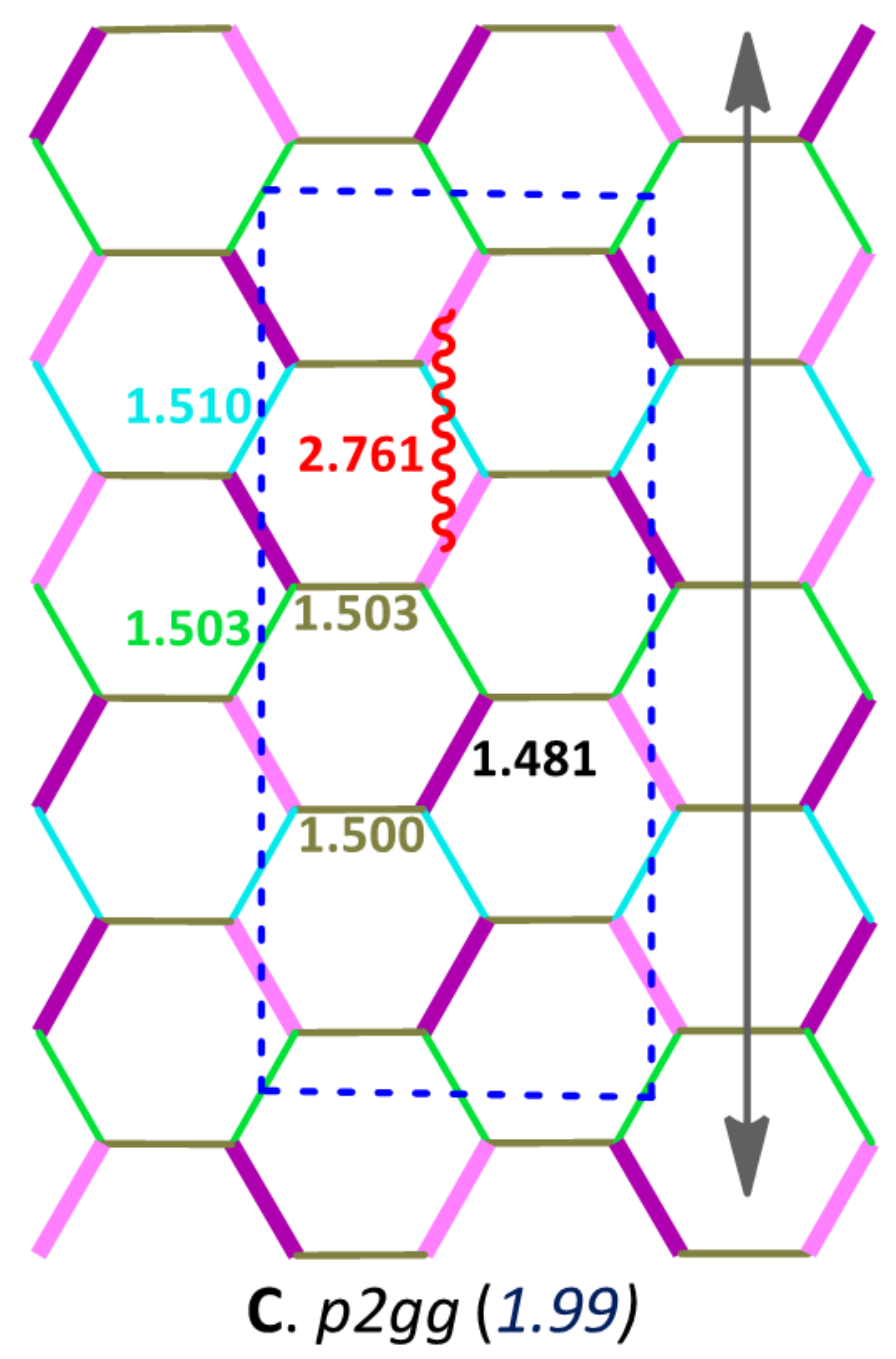


Lattice parameters $(\AA ̊) \quad$ Cell Angles

$$
\begin{array}{ll}
a=4.460 & \alpha=90.00 \\
b=25.000 & \beta=90.83 \\
c=5.112 & \gamma=90.00
\end{array}
$$

\section{Isomer E}

\begin{tabular}{|c|c|c|c|}
\hline C & -0.013220 & 0.966355 & 0.750000 \\
\hline C & 0.013220 & -0.966355 & 1.250000 \\
\hline C & 0.013220 & 1.466355 & -0.250000 \\
\hline C & -0.013220 & -0.466355 & -0.7 \\
\hline C & 0.039413 & 0.201612 & -1.750000 \\
\hline C & -0.039413 & -0.201612 & -1.250000 \\
\hline$C$ & -0.039413 & 0.701612 & 2.250000 \\
\hline C & 0.039413 & 0.298388 & 1.750000 \\
\hline 0 & -0.078212 & 0.941525 & 0.750000 \\
\hline 0 & 0.078212 & -0.941525 & 1.250000 \\
\hline 0 & 0.078212 & 1.441525 & -0.250000 \\
\hline 0 & -0.078212 & -0.44152 & -0.7 \\
\hline
\end{tabular}

Element Fractional coordinates of atoms

$$
\text { u } \quad v \quad w
$$

Lattice parameters $(\AA \AA) \quad$ Cell Angles

$$
\begin{array}{ll}
a=25.000 & \alpha=90.00 \\
b=8.169 & \beta=90.00 \\
c=2.586 & \gamma=90.00
\end{array}
$$



E. $p 2 m g(4.11)$

Isomer F

Element Fractional coordinates of atoms

\begin{tabular}{crcc} 
& $\mathbf{u}$ & $\mathbf{v}$ & $\mathbf{w}$ \\
\hline $\mathrm{C}$ & 0.500000 & 0.832502 & -0.007595 \\
$\mathrm{C}$ & -0.500000 & -0.832502 & -0.007595 \\
$\mathrm{C}$ & 0.000000 & 1.332502 & 0.007595 \\
$\mathrm{C}$ & 1.000000 & -0.332502 & 0.007595 \\
$\mathrm{O}$ & 0.000000 & 0.500000 & 0.068069 \\
$\mathrm{O}$ & 0.500000 & 1.000000 & -0.068069
\end{tabular}

Lattice parameters(Å) Cell Angles

$$
\begin{array}{ll}
a=2.589 & \alpha=90.00 \\
b=4.436 & \beta=90.00 \\
c=25.000 & \gamma=90.00
\end{array}
$$

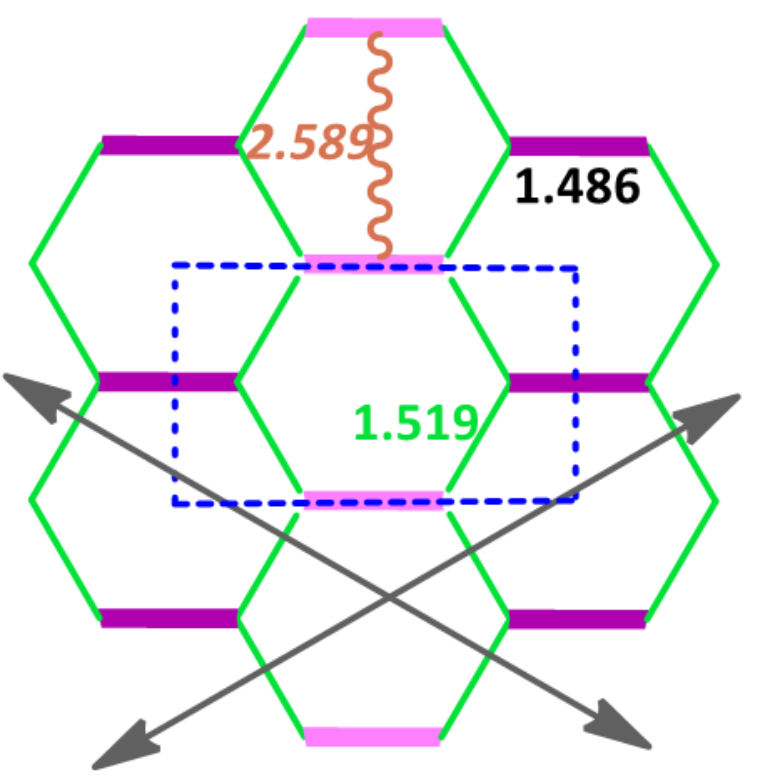

F. $c 2 m m(4.30)$ 


\section{Isomer G}

Element Fractional coordinates of atoms

\begin{tabular}{lrrr} 
& \multicolumn{2}{c}{ u } & v \\
\hline C & 0.462129 & 0.298647 & 0.008588 \\
C & 0.292137 & -0.036365 & 0.009577 \\
C & 0.298647 & 0.462129 & -0.008588 \\
$C$ & -0.036365 & 0.292137 & -0.009577 \\
C & 0.798647 & -0.037871 & 0.008588 \\
$C$ & 0.463635 & -0.207863 & 0.009577 \\
C & 0.962129 & -0.201353 & -0.008588 \\
$C$ & 0.792137 & -0.536365 & -0.009577 \\
$O$ & 0.359889 & 0.139535 & 0.068455 \\
$O$ & 0.139535 & 0.359889 & -0.068455 \\
$O$ & 0.639535 & -0.140111 & 0.068455 \\
$O$ & 0.859889 & -0.360465 & -0.068455
\end{tabular}

Lattice parameters $(\AA \AA) \quad$ Cell Angles

$$
\begin{array}{ll}
a=5.149 & \alpha=90.00 \\
b=5.149 & \beta=90.00 \\
c=25.000 & \gamma=120.82
\end{array}
$$

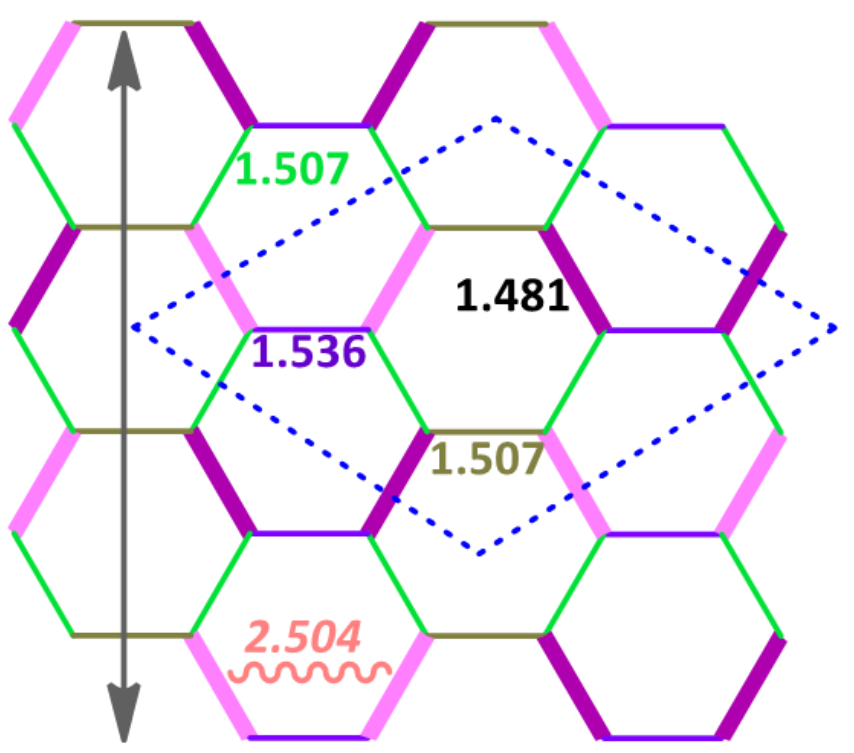

G. $p 2 m g(4.43)$

\section{Isomer H}

Element Fractional coordinates of atoms

u $\quad \mathbf{w}$

\begin{tabular}{cccc}
\hline$C$ & 0.663132 & 0.007648 & 0.166853 \\
C & -0.663132 & 0.007648 & 0.333147 \\
C & -0.663132 & -0.007648 & -0.166853 \\
C & 0.663132 & -0.007648 & 0.666853 \\
$O$ & 0.500000 & -0.067822 & -0.250000 \\
$O$ & -0.500000 & 0.067822 & 0.250000
\end{tabular}

\section{Lattice parameters $(\AA) \quad$ Cell Angles}

$$
\begin{array}{ll}
a=2.637 & \alpha=90.00 \\
b=25.000 & \beta=121.44 \\
c=5.131 & \gamma=90.00
\end{array}
$$

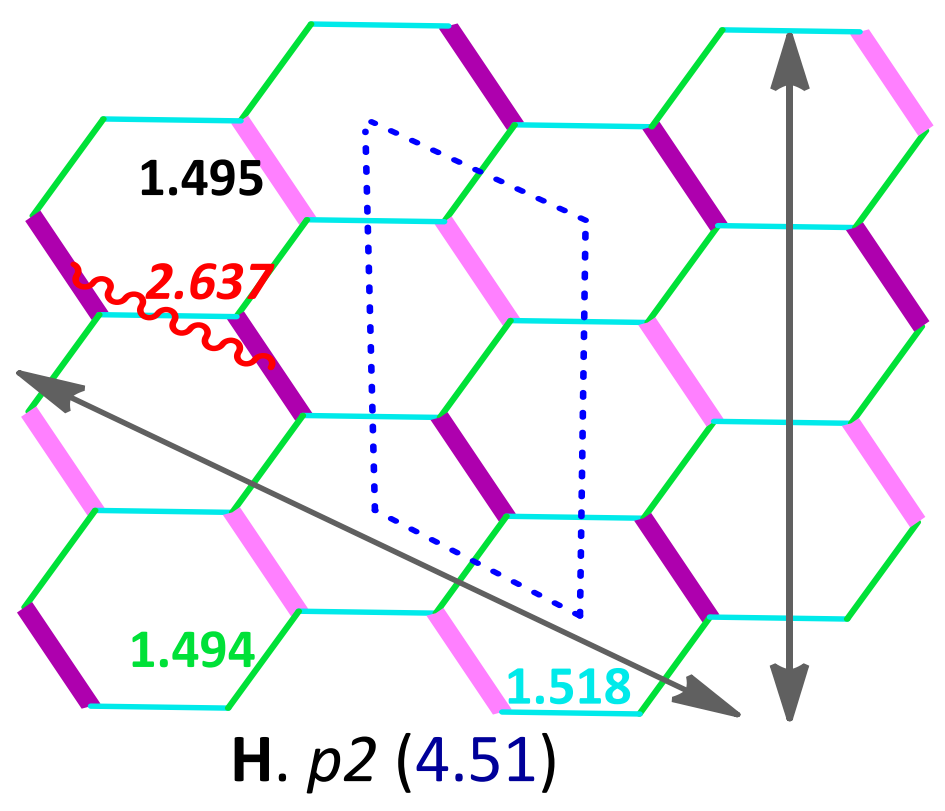


Isomer I

Element Fractional coordinates of atoms

\begin{tabular}{cccc} 
& \multicolumn{2}{c}{$\mathbf{u}$} & $\mathbf{w}$ \\
\hline $\mathrm{C}$ & 0.500795 & -0.024544 & -0.661291 \\
$\mathrm{C}$ & 0.994423 & -0.016359 & -0.084072 \\
$\mathrm{C}$ & -0.500795 & -0.024544 & 1.161291 \\
$\mathrm{C}$ & -0.994423 & -0.016359 & 0.584072 \\
$\mathrm{C}$ & -0.500795 & 0.024544 & 0.661291 \\
$\mathrm{C}$ & -0.994423 & 0.016359 & 0.084072 \\
$\mathrm{C}$ & 0.500795 & 0.024544 & -0.161291 \\
$\mathrm{C}$ & 0.994423 & 0.016359 & 0.415928 \\
$\mathrm{O}$ & 0.254269 & 0.079758 & -0.660221 \\
$\mathrm{O}$ & -0.254269 & 0.079758 & 1.160221 \\
$\mathrm{O}$ & -0.254269 & -0.079758 & 0.660221 \\
$\mathrm{O}$ & 0.254269 & -0.079758 & -0.160221
\end{tabular}

Lattice parameters(Å) Cell Angles

$$
\begin{array}{ll}
a=8.927 & \alpha=90.00 \\
b=25.000 & \beta=162.82 \\
c=8.542 & \gamma=90.00
\end{array}
$$

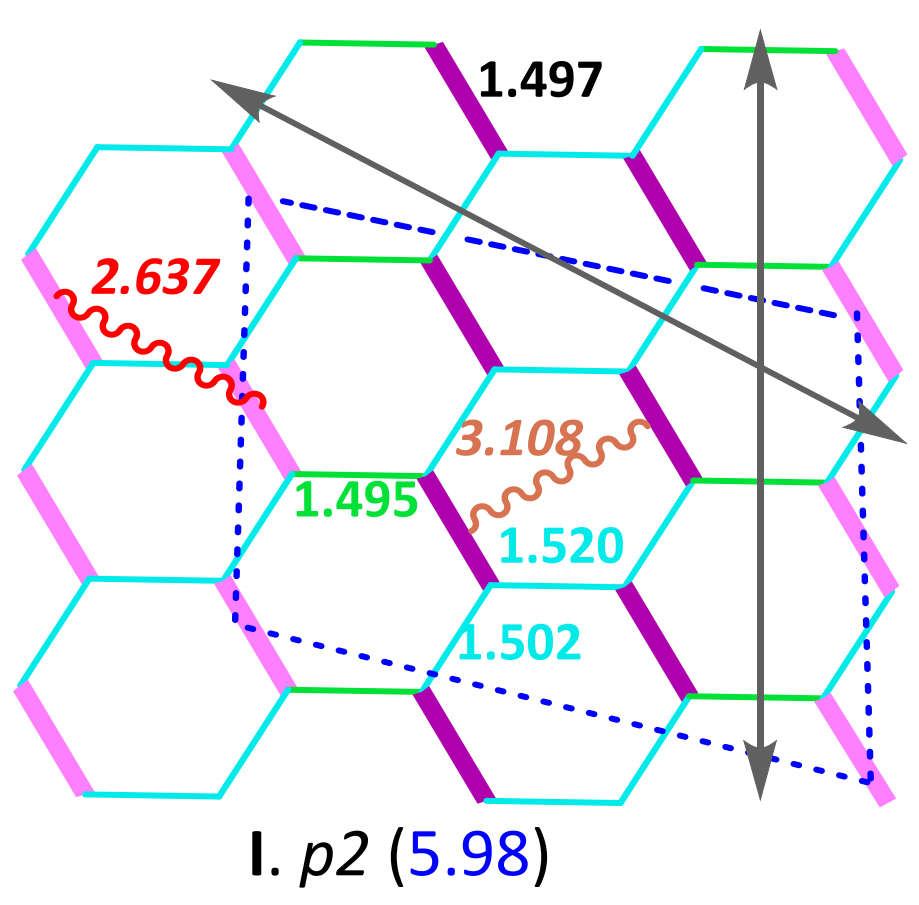

Isomer J

Element Fractional coordinates of atoms

u $\quad \mathbf{w}$

\begin{tabular}{lrrr}
\hline$C$ & 0.008551 & 0.246264 & -0.583581 \\
$C$ & -0.008551 & -0.246264 & -0.083581 \\
$C$ & -0.008551 & 0.246264 & 1.083581 \\
$C$ & 0.008551 & -0.246264 & 0.583581 \\
$O$ & 0.068849 & 0.000000 & -0.500000 \\
$O$ & -0.068849 & 0.000000 & 0.000000
\end{tabular}

Lattice parameters(Å) Cell Angles

$$
\begin{array}{ll}
a=25.000 & \alpha=90.00 \\
b=2.632 & \beta=90.00 \\
c=4.375 & \gamma=90.00
\end{array}
$$

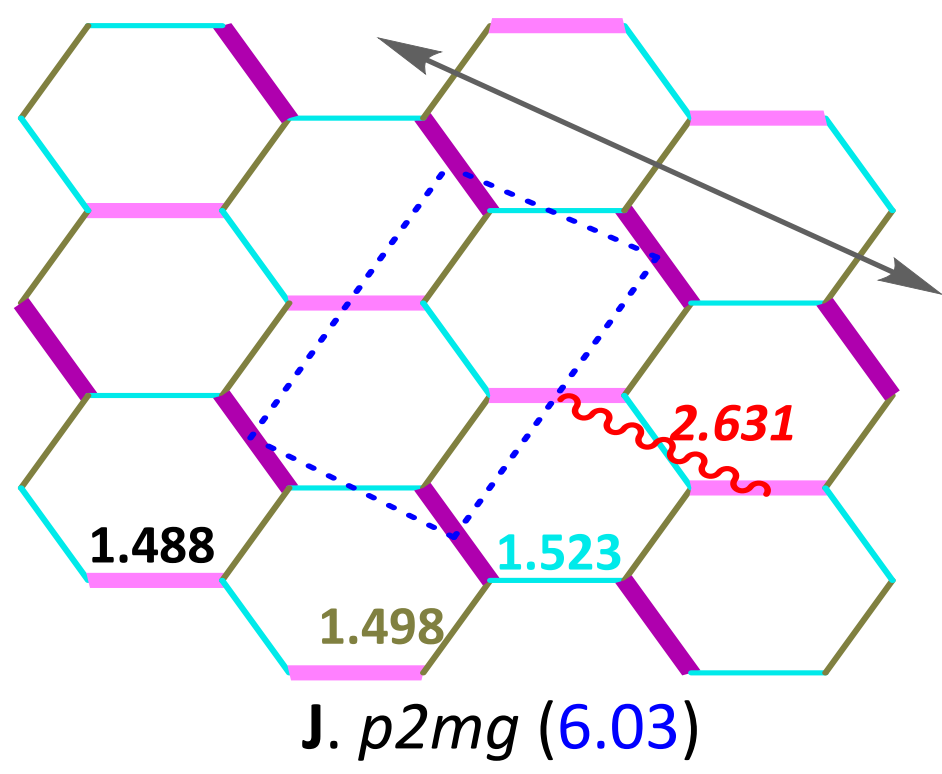




\section{Isomer K}

Element Fractional coordinates of atoms

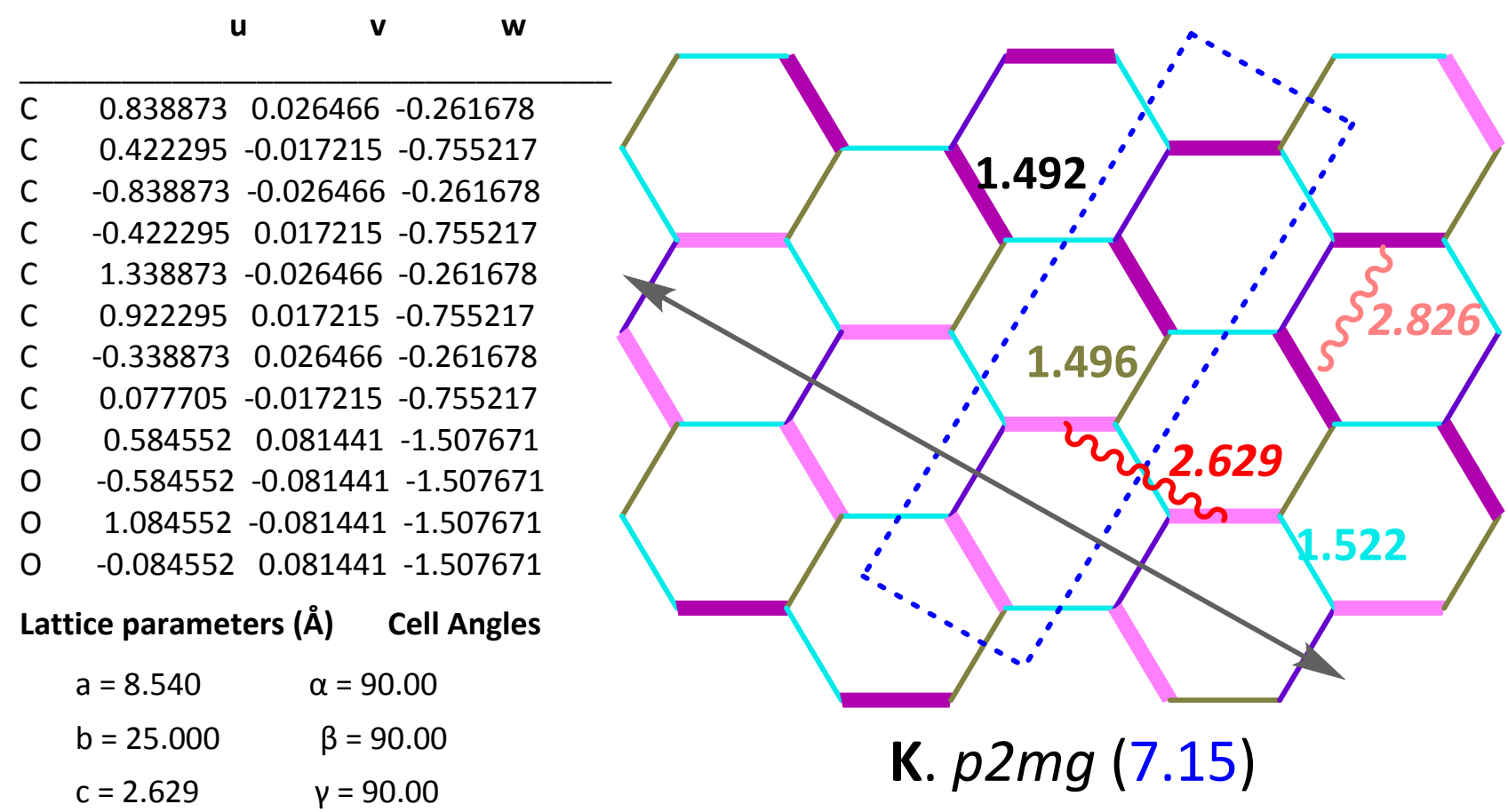

Isomer L

Element Fractional coordinates of atoms

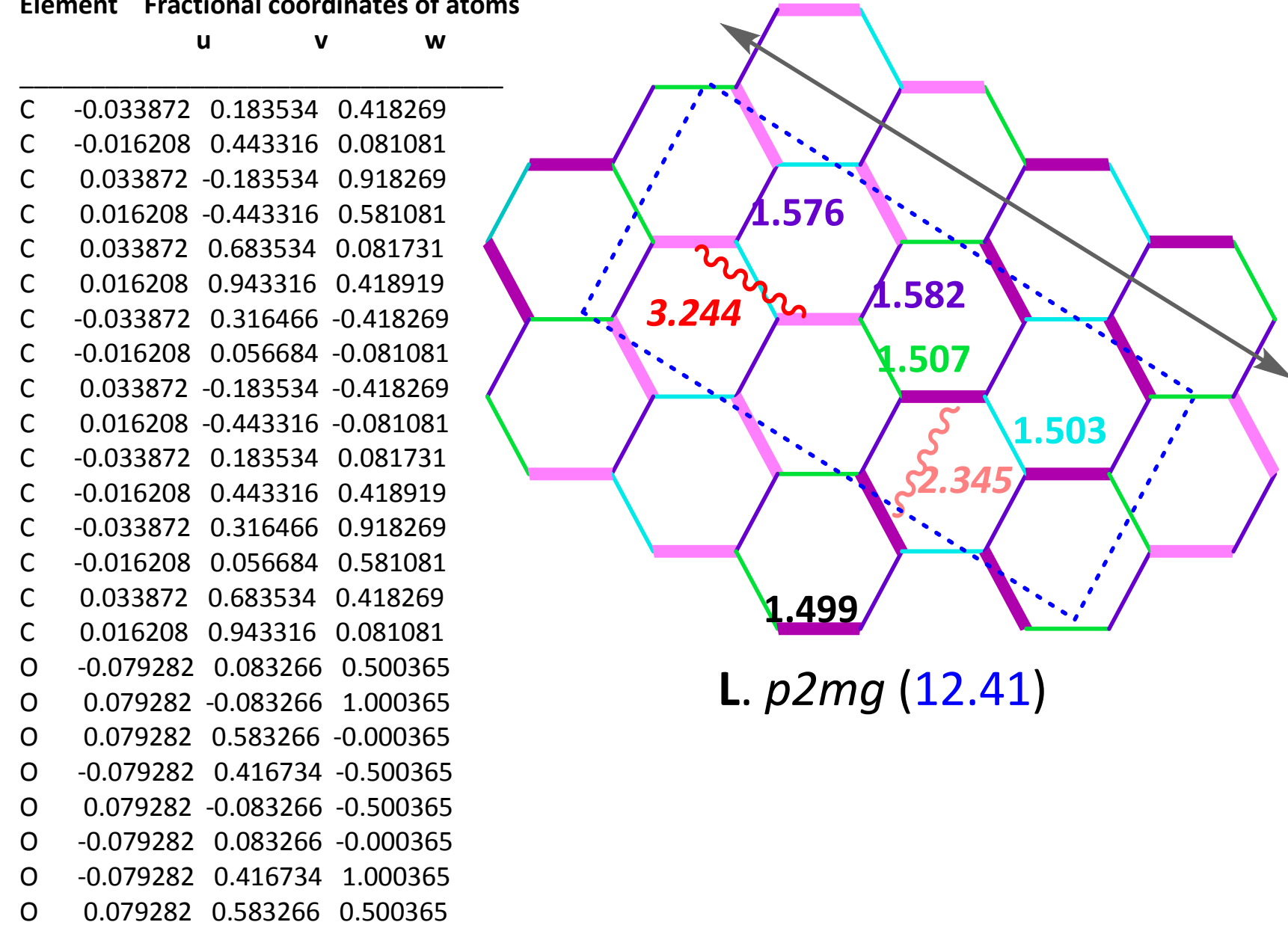


Lattice parameters (Å) Cell Angles

$$
\begin{array}{ll}
a=25.000 & \alpha=90.00 \\
b=9.728 & \beta=90.00 \\
c=4.682 & \gamma=90.00
\end{array}
$$

\section{Isomer M}

Element Fractional coordinates of atoms

v w

$\begin{array}{llll}\text { C } & -0.008053 & 0.376451 & 0.418740\end{array}$

C $\quad 0.008053-0.3764510 .918740$

C $\quad 0.008053 \quad 0.876451 \quad 0.081260$

C $\quad-0.008053 \quad 0.123549-0.418740$

C $0.008053-0.376451-0.418740$

C $\quad-0.008053 \quad 0.3764510 .081260$

C $\quad-0.008053 \quad 0.1235490 .918740$

C $\quad 0.0080530 .876451 \quad 0.418740$

$\begin{array}{llll}\text { O } & -0.067292 & 0.250000 & 0.500000\end{array}$

$\begin{array}{llll}0 & 0.067292 & -0.250000 & 1.000000\end{array}$

O $0.067292-0.250000-0.500000$

$\begin{array}{lllll}0 & -0.067292 & 0.250000 & 0.000000\end{array}$

Lattice parameters ( $(\AA) \quad$ Cell Angles

$$
\begin{array}{ll}
a=25.000 & \alpha=90.00 \mathrm{~s} \\
b=5.097 & \beta=90.00 \\
c=4.679 & \gamma=90.00
\end{array}
$$

\section{Isomer N}

Element Fractional coordinates of atoms

\begin{tabular}{crrc} 
& u & $\mathbf{v}$ & w \\
\hline$C$ & 1.336703 & -0.663297 & 0.002439 \\
$C$ & -1.336703 & 0.663297 & 0.002439 \\
O & 1.500000 & -0.500000 & 0.043724
\end{tabular}

\section{Lattice parameters(Å) Cell Angles}

$$
\begin{array}{ll}
a=2.664 & \alpha=90.00 \\
b=2.664 & \beta=90.00 \\
c=25.000 & \gamma=58.94
\end{array}
$$

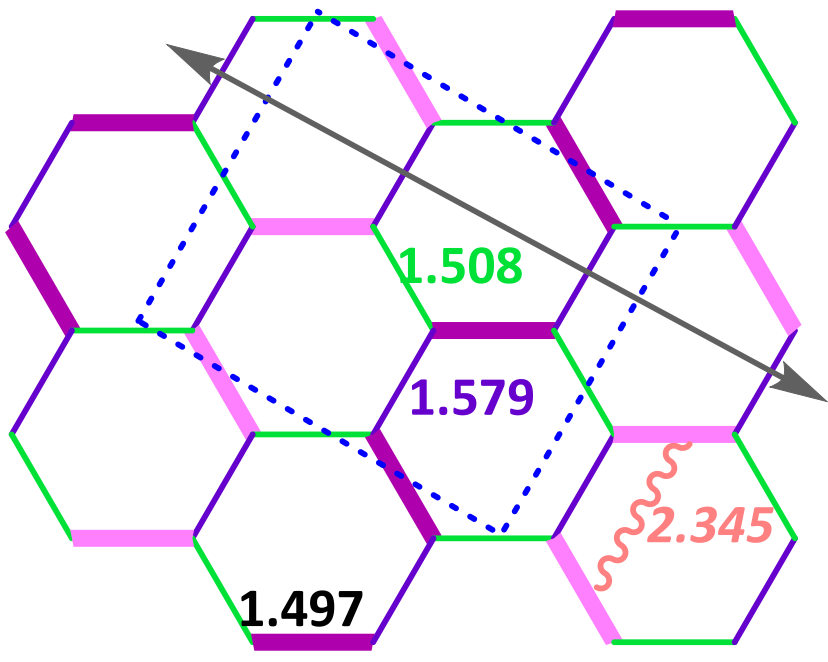

M. $p 2 m g(12.53)$

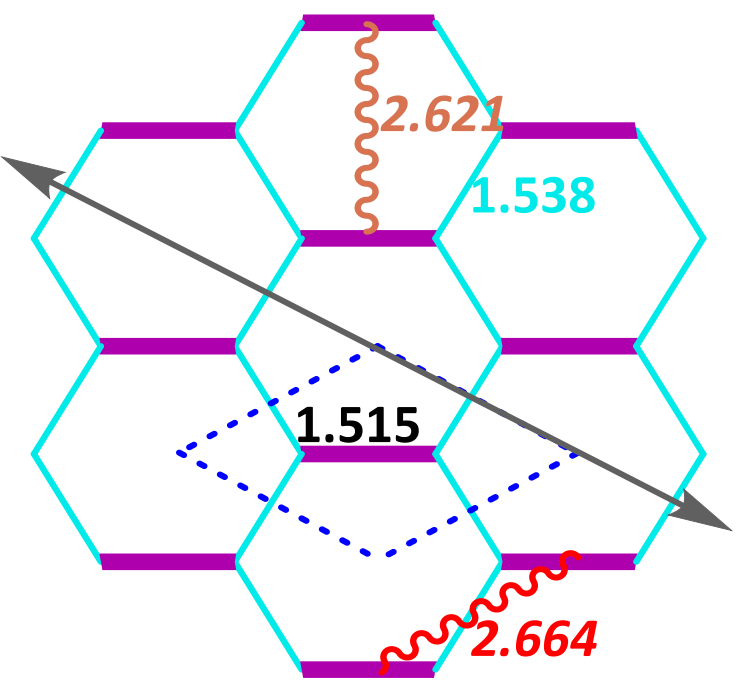

N. $c 2 m m(16.67)$ 


\section{Isomer $\mathbf{O}$}

Element Fractional coordinates of atoms

u

v

w

C $\quad 0.580980 \quad 0.747971 \quad-0.998554$

$\begin{array}{llll}\text { C } & -0.580980 & -0.747971 & -0.998554\end{array}$

C $\quad 1.080980-0.747971-0.998554$

C $\quad-0.080980 \quad 0.747971-0.998554$

O $\quad 0.500000 \quad 0.500000-1.053210$

O $1.000000-0.500000-1.053210$

Lattice parameters(Å) Cell Angles

$$
\begin{array}{ll}
a=4.687 & \alpha=90.00 \\
b=2.640 & \beta=90.00 \\
c=25.000 & \gamma=90.00
\end{array}
$$

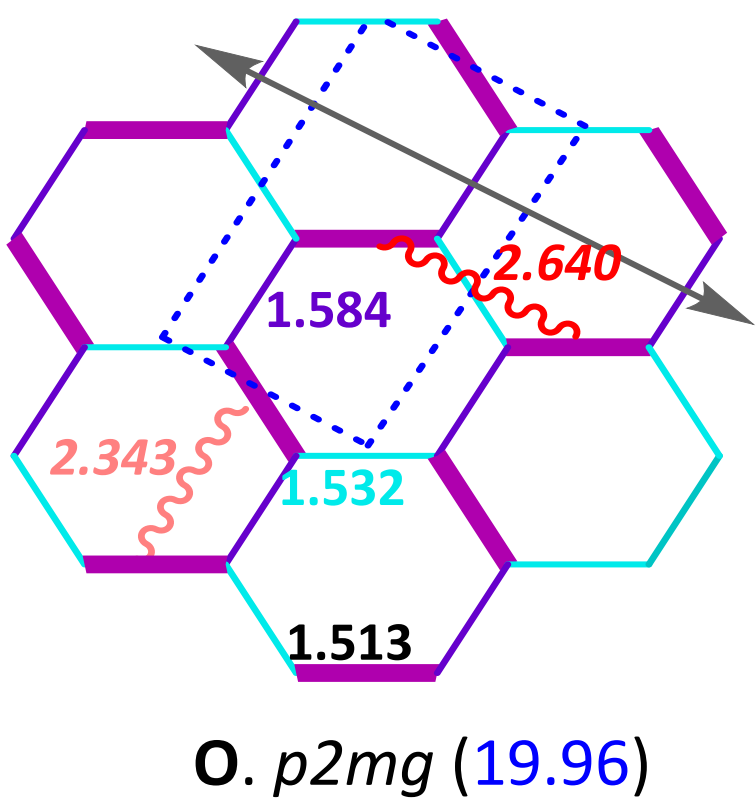

Isomer P

Element Fractional coordinates of atoms

\begin{tabular}{lrrr} 
& \multicolumn{2}{c}{$\mathbf{u}$} & $\mathbf{w}$ \\
& & & \\
\hline C & -1.000000 & -0.335070 & 0.001540 \\
C & 0.335070 & -0.664930 & 0.001540 \\
C & 0.664930 & 1.000000 & 0.001540 \\
$C$ & 1.000000 & 0.335070 & 0.001540 \\
$C$ & -0.335070 & 0.664930 & 0.001540 \\
$C$ & -0.664930 & -1.000000 & 0.001540 \\
O & -0.500000 & -0.500000 & 0.041030 \\
O & 0.500000 & 0.000000 & 0.041030 \\
O & 0.000000 & 0.500000 & 0.041030
\end{tabular}

\section{Lattice parameters( $(\AA) \quad$ Cell Angles}

$$
\begin{array}{ll}
a=4.687 & \alpha=90.00 \\
b=4.687 & \beta=90.00 \\
c=25.000 & \gamma=120.00
\end{array}
$$

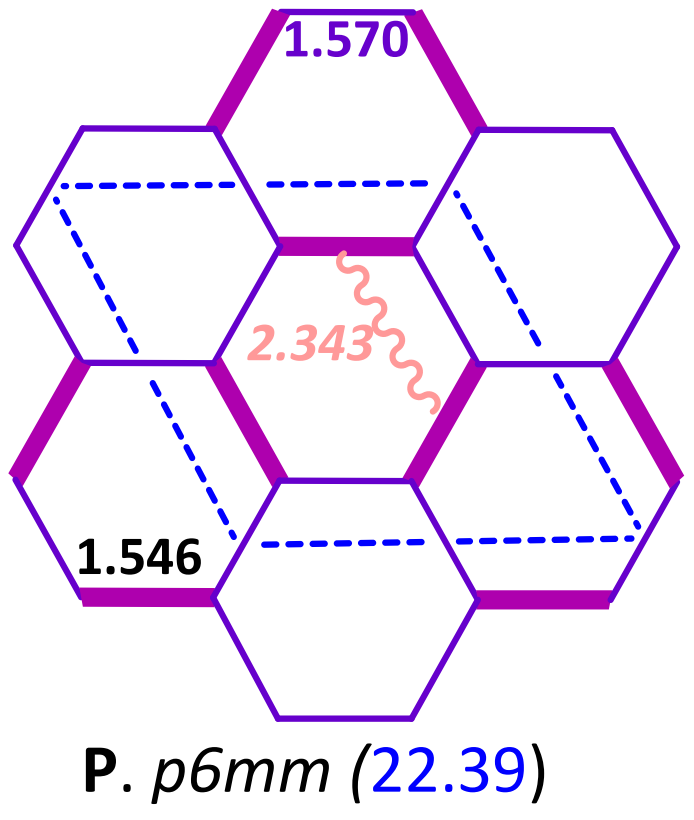


Table S1 Geometrical details of the various optimized $\mathrm{C}_{2} \mathrm{O}$ sheets studied at dispersion corrected GGA/PBE.

\section{*Total number of epoxide interactions present per unit cell}

\begin{tabular}{|c|c|c|c|c|c|c|c|c|c|c|c|c|c|c|}
\hline \multirow[t]{3}{*}{ Isomers } & \multirow[t]{3}{*}{$z$} & \multirow[t]{3}{*}{ Total $^{*}$} & \multicolumn{4}{|c|}{1,3 exo $C-C$} & \multirow{3}{*}{$\begin{array}{c}\mathbf{1 , 4} \\
\text { intra } \\
\text { Cis } \\
0 . . .0 \\
\end{array}$} & \multirow{3}{*}{\begin{tabular}{|c|}
1,3 \\
Intra \\
Cis $0 \ldots 0$ \\
\end{tabular}} & \multirow{3}{*}{\begin{tabular}{|c|}
$\mathbf{1 , 3}$ \\
inter \\
Cis \\
$0 . . .0$ \\
\end{tabular}} & \multirow{3}{*}{\begin{tabular}{|c|} 
C-O \\
Distance \\
\end{tabular}} & \multirow{3}{*}{\begin{tabular}{|c|} 
Splaying \\
0
\end{tabular}} & \multirow{3}{*}{$\begin{array}{c}\text { C-C } \\
\text { Epoxy }\end{array}$} & \multirow{3}{*}{$\begin{array}{c}\begin{array}{c}\text { Pyram. } \\
\text { Angle }\end{array} \\
\mathrm{C}\end{array}$} & \multirow{3}{*}{$\begin{array}{c}\text { Band } \\
\text { gap } \\
(e V)\end{array}$} \\
\hline & & & \multicolumn{2}{|c|}{ intra } & \multicolumn{2}{|c|}{ inter } & & & & & & & & \\
\hline & & & cis & trans & cis & trans & & & & & & & & \\
\hline A & 4 & 8 & --- & $1.500(4)$ & --- & $1.504(4)$ & --- & & & 1.447 & $\mathrm{NO}$ & 1.475 & 6.07 & 4.472(D) \\
\hline B & 4 & 8 & & & $1.505(4)$ & $1.501(4)$ & & & $2.911(4)$ & 1.440 & \begin{tabular}{|l|} 
Lateral \\
\end{tabular} & 1.483 & 5.24 & $4.616(I)$ \\
\hline C & 8 & 16 & & $\begin{array}{l}1.503(4) \\
1.500(4)\end{array}$ & $1.510(4)$ & $1.503(4)$ & $2.670(2)$ & & $2.761(4)$ & $\begin{array}{l}1.458 \\
1.436\end{array}$ & \begin{tabular}{|c|} 
Axial \\
Lateral
\end{tabular} & 1.481 & $\begin{array}{c}6.1 \\
3.01\end{array}$ & $4.233(D)$ \\
\hline D & 4 & 8 & & & $1.506(2)$ & $1.512(6)$ & $2.670(2)$ & & $2.753(4)$ & $\begin{array}{l}1.451 \\
1.436\end{array}$ & \begin{tabular}{|c|} 
Axial \\
Lateral
\end{tabular} & 1.486 & $\begin{array}{c}4 \\
2.83 \\
\end{array}$ & $14(D)$ \\
\hline$E$ & 4 & 8 & & & $1.515(4)$ & $1.519(4)$ & $2.586(4)$ & & $3.368(4)$ & $\begin{array}{l}1.444 \\
1.433 \\
\end{array}$ & \begin{tabular}{|c|} 
Axial \\
Lateral
\end{tabular} & 1.486 & $\begin{array}{c}5.32 \\
5.9 \\
\end{array}$ & 63(D) \\
\hline$F$ & 2 & 4 & & & & $1.519(4)$ & $2.589(2)$ & & & 1.438 & NO & 1.486 & 5.46 & $3.225(\mathrm{D})$ \\
\hline G & 4 & 8 & $1.536(2)$ & $1.507(2)$ & & $1.507(4)$ & & $2.504(2)$ & & $\begin{array}{l}1.453 \\
1.432\end{array}$ & \begin{tabular}{|c|} 
Axial \\
Lateral
\end{tabular} & 1.481 & $\begin{array}{c}7.3 \\
2.33 \\
\end{array}$ & $4.174(I)$ \\
\hline H & 2 & 4 & & & $1.518(2)$ & $1.494(2)$ & & & $2.637(2)$ & 1.442 & NO & 1.495 & 1.41 & 4.637(D) \\
\hline 1 & 6 & 12 & & & $\begin{array}{l}1.502(4) \\
1.520(4)\end{array}$ & $1.495(4)$ & $3.108(2)$ & & $2.637(4)$ & $\begin{array}{l}1.438 \\
1.445\end{array}$ & \begin{tabular}{|c|} 
Axial \\
Lateral
\end{tabular} & 1.497 & $\begin{array}{l}1.67 \\
1.63\end{array}$ & 4.454(D) \\
\hline $\mathrm{J}$ & 2 & 4 & & $1.498(2)$ & $1.523(2)$ & & & & $2.631(2)$ & 1.446 & NO & 1.488 & 1.77 & 4.499(D) \\
\hline K & 4 & 8 & $1.518(2)$ & $1.496(2)$ & $1.522(4)$ & & & $2.826(2)$ & $2.629(4)$ & $\begin{array}{l}1.445 \\
1.437\end{array}$ & $\begin{array}{c}\text { Axial } \\
\text { Lateral }\end{array}$ & 1.492 & $\begin{array}{l}1.61 \\
2.07\end{array}$ & $4.345(I)$ \\
\hline $\mathbf{L}$ & 8 & 16 & $\begin{array}{l}1.582(4) \\
1.576(4)\end{array}$ & & $1.503(4)$ & $1.507(4)$ & & $2.345(4)$ & $3.244(4)$ & $\begin{array}{l}1.428 \\
1.422\end{array}$ & \begin{tabular}{|c|} 
Axial \\
Lateral
\end{tabular} & 1.499 & $\begin{array}{l}2.09 \\
1.67\end{array}$ & $3.776(\mathrm{D})$ \\
\hline$M$ & 4 & 8 & $1.579(4)$ & & & $1.508(4)$ & & $2.345(4)$ & & 1.426 & NO & 1.497 & 1.58 & $3.610(D)$ \\
\hline $\mathrm{N}$ & 1 & 2 & & & $1.538(2)$ & & 2.621(1) & & $2.664(2)$ & 1.432 & $\mathrm{NO}$ & 1.515 & 0 & \begin{tabular}{|l}
$3.324(I)$ \\
\end{tabular} \\
\hline 0 & 2 & 4 & $1.584(2)$ & & $1.532(2)$ & & $2.343(2)$ & & $2.640(2)$ & 1.42 & NO & 1.513 & 0 & $4.065(D)$ \\
\hline $\mathbf{P}$ & 3 & 6 & $1.570(6)$ & & & & & $2.343(6)$ & & 1.408 & NO & 1.546 & 0 & $2.947(D)$ \\
\hline
\end{tabular}

